\title{
Collective plasmonic modes in two-dimensional periodic arrays of metal nanoparticles
}

\author{
Yu-Rong Zhen, Kin Hung Fung, and C. T. Chan \\ Physics Department, Hong Kong University of Science and Technology, Clear Water Bay, Kowloon, Hong Kong \\ (Received 28 March 2008; revised manuscript received 28 May 2008; published 11 July 2008)
}

\begin{abstract}
We investigate the collective plasmonic modes of metal nanoparticles in periodic two-dimensional (2D) arrays within a point-dipole description. The dynamic dispersion relations of the $2 \mathrm{D}$ arrays are obtained through a method which gives an effective polarizability describing the collective response of a system. Both the dispersion relations and mode qualities are simultaneously related to the imaginary part of the effective polarizability, which has contributions from the single-particle response as well as the interparticle coupling. The transverse long-range dipolar interaction is dominated by a wave term together with a purely geometrical constant representing the static geometrical contribution to resonant frequencies. As concrete examples, we considered small Ag spheres arranged in a square lattice. We find that inside the light cone, the transverse quasimode has a reasonably high mode quality, while the two in-plane modes show significant radiation damping. Near the light line, we observe strong coupling with free photons for the bands of the transverse mode and the transverse in-plane mode, and the longitudinal in-plane mode exhibits a negative group velocity inside the light cone. Vanishing group velocities in the light cone for all the quasimodes are found to be intrinsic properties of the 2D metal nanosphere dense arrays.
\end{abstract}

DOI: 10.1103/PhysRevB.78.035419

PACS number(s): 78.67.Bf, 71.15.Ap, 73.22.Lp, 02.30.Lt

\section{INTRODUCTION}

The optical properties of clusters of metal nanoparticles have attracted much interest because of their plausible nanophotonic applications such as optical waveguides, ${ }^{1-5}$ biosensors, ${ }^{6,7}$ and subwavelength imaging. ${ }^{8,9}$ The plasmonic resonance in noble-metal particles, particularly nanospheres, can often be modeled adequately as a dipolar resonance as long as the interparticle spacing is large enough so that the high-order resonances are not important. In those systems, the collective behavior of a cluster of particles can be directly modeled with coupled-dipole equations. Based on the coupled-dipole model, the plasmonic modes of metal nanoparticles in one-dimensional (1D) periodic systems, such as single chain ${ }^{2,8,10-16}$ and double chains, ${ }^{16}$ have been extensively investigated. At the same time, there are also some interesting experimental ${ }^{17,18}$ and theoretical ${ }^{9,14,19-22}$ results on the two-dimensional (2D) arrays of nanoparticles or dipolar scatters.

A popular approach to treat those systems is to obtain the eigenmodes, i.e., the dispersion $(\omega-k)$ relations by assuming that the system under investigation is a closed system (no coupling to the free photons and dissipative environment). Once the conventional time dependence $e^{-i \omega t}$ is adopted, in the absence of driving field, the oscillating frequency $\omega$ of an actual system is a complex quantity with an imaginary part satisfying $\operatorname{Im}(\omega) \leq 0$ because as an actual system it will be coupled to either the dissipative environment or free photons, leading to decaying oscillations in time. As a result of the negative imaginary part of $\omega$, there is a divergence in the lattice sum of dynamic dipolar Green's function. ${ }^{11}$ Usually, there are three methods to avoid such difficulty. The first method is to model the system approximately as a "closed" system, as in the quasistatic approximation ${ }^{2,11}$ where $\operatorname{Im}(\omega)$ $=0$. Thus the dispersion relations can be obtained in closed form in $1 \mathrm{D}$ system. The second approach is to use a finite number of particles to emulate an infinite periodic structure, ${ }^{11}$ and dispersion relations are obtained by searching the loci of global minima of the determinant of a coupling matrix. The third approach is to evaluate the sums in the $\operatorname{Im}(\omega) \geq 0$ half-plane and then carry out analytic continuation to the $\operatorname{Im}(\omega) \leq 0$ half-plane. ${ }^{15,23}$ The above approaches are applicable in 1D system but they have their own limitations. The quasistatic approximation cannot account for the coupling between the free-propagating wave in the ambient medium and the eigenmodes of the system. The finite-size treatment in one-dimensional chain ${ }^{11}$ requires the evaluation of the determinant of the coupling matrix, whose dimension $N$ is proportional to the number of particles, as a function of the complex frequency at a given spatial wave vector. In addition, the pole searching is not direct but should be accompanied with a tracing from the quasistatic limit because the number of poles is larger than the number of dipoles. ${ }^{15}$ The evaluation of determinant typically requires an $O\left(N^{3}\right)$ algorithm and $O(N)$ times of evaluation are needed to find out the $N$ poles of the complex function. The finite-size treatment has a complexity of $O\left(N^{4}\right)$ and becomes computationally demanding in two-dimensional arrays because of increased dimension of the coupling matrix. Lastly, while the analytic continuation method is realizable in infinite 1D chain, it becomes complicated in infinite $2 \mathrm{D}$ arrays. The above difficulties may explain the lack of full study of dispersion relations for the collective modes of periodic dipoles in infinite 2D arrays, while there are many such studies for 1D chains.

More efficient approaches are, in fact, available to obtain the dispersion relations and quality factors simultaneously ${ }^{16}$ and have been applied to 1D systems. For an open system, it is more appropriate (both from a computational point of view and from the physics point of view) to consider the system as a driven system instead of a system without external driving. In the presence of incident wave, an effective polarizability can be introduced within the framework of spectral decomposition $^{24,25}$ to describe the response from not only material properties but also collective coupling in a specific 
geometrical structure. We will apply such an approach to two-dimensional dipole arrays.

In this paper, we will focus on plasmonic dispersions of nanospheres in infinite 2D periodic arrays. In Sec. II we consider the point-dipole model which has been shown to give a very good description of small metal particles ${ }^{26}$ as long as $a \leq d / 3$, where $a$ is the sphere's radius and $d$ is the lattice constant. We first discuss the dispersion relations within the quasistatic dipolar approximation in Sec. III. In Sec. IV, we show the dynamic dispersion relations and quality factors simultaneously, in which the retardation effect is also included. Numerical results are given for a specific example that describes silver nanospheres arranged in infinite square lattice, followed by a discussion and a brief conclusion.

\section{COUPLED-DIPOLE EQUATIONS}

The local electromagnetic field at the position of a dipole consists of the incident field and the field radiated by all the other dipoles. On the local dipole the local field $\mathbf{E}_{\text {local }}$ induces a dipole moment $\mathbf{p}_{i}$, which is itself a radiation source to other dipoles. If the time dependence is assumed to be $e^{-i \omega t}$ and the polarizability $\alpha$ is assumed to be isotropic and identical for every dipole, then for $i$ th dipole, we have the following coupled-dipole equation, ${ }^{27}$

$$
\mathbf{p}_{i}=\alpha\left[\mathbf{E}_{\mathrm{inc}, i}+\sum_{j \neq i} \mathbf{G}\left(\mathbf{R}_{i}, \mathbf{R}_{j}\right) \mathbf{p}_{j}\right],
$$

where

$$
\begin{aligned}
\mathbf{G}\left(\mathbf{R}_{i}, \mathbf{R}_{j}\right) \mathbf{p}_{j}= & {\left[\left(1-\frac{\left.\mathrm{i} \omega \mathrm{r}_{i j}\right)}{c}\right) \frac{3\left(\mathbf{n}_{i j} \cdot \mathbf{p}_{j}\right) \mathbf{n}_{i j}-\mathbf{p}_{j}}{r_{i j}^{3}}\right.} \\
& \left.+\frac{\omega^{2}}{c^{2}} \frac{\mathbf{p}_{j}-\left(\mathbf{n}_{i j} \cdot \mathbf{p}_{j}\right) \mathbf{n}_{i j}}{r_{i j}}\right] \exp \left(\frac{i \omega \mathbf{r}_{i j}}{c}\right) .
\end{aligned}
$$

Here $\mathbf{E}_{\mathrm{inc}, i}$ is the incident field at $i$ th dipole, $\mathbf{G}\left(\mathbf{R}_{i}, \mathbf{R}_{j}\right) \mathbf{p}_{j}$ represents the field radiated by $j$ th dipole at the position of $i$ th dipole, $\mathbf{R}$ is the position of a dipole, $\mathbf{r}_{i j}=\mathbf{R}_{i}-\mathbf{R}_{j}, \mathbf{n}_{i j}=\mathbf{r}_{i j} / r_{i j}$ is a unit vector parallel to $\mathbf{r}_{i j}$, and $c$ is the speed of light. Equation (2.1) can be rewritten as

$$
\frac{1}{\alpha} \mathbf{p}_{i}-\sum_{j \neq i} \mathbf{G}\left(\mathbf{R}_{i}, \mathbf{R}_{j}\right) \mathbf{p}_{j}=\mathbf{E}_{\mathrm{inc}, i} .
$$

For periodic systems, we apply the periodic boundary conditions,

$$
\begin{aligned}
\mathbf{p}_{i} & =\mathbf{p} \exp \left(i \mathbf{k}_{\mathbf{B}} \cdot \mathbf{R}_{i}\right), \\
\mathbf{E}_{\text {inc }, i} & =\mathbf{E}_{\text {inc }} \exp \left(i \mathbf{k}_{\mathbf{B}} \cdot \mathbf{R}_{i}\right),
\end{aligned}
$$

where $\mathbf{k}_{\mathbf{B}}$ is the Bloch wave vector, and the form of external field [Eq. (2.5)] is chosen to excite one particular eigenmode with Bloch vector $\mathbf{k}_{\mathbf{B}}$. We put Eqs. (2.4) and (2.5) into Eq. (2.3) and have

$$
\mathbf{M p}=\mathbf{E}_{\text {inc }},
$$

where $\mathbf{E}_{\text {inc }}$ is a vector whose components are the electric fields of external incident wave, $\mathbf{M}$ is a $3 \times 3$ matrix operating on three-dimensional (3D) vector $\mathbf{p}$,

$$
\mathbf{M}=\frac{1}{\alpha} \mathbf{I}-\boldsymbol{\beta},
$$

I is a unit matrix, and $\boldsymbol{\beta}$ is a dyadic of lattice sum of dipolar Green's function,

$$
\boldsymbol{\beta}=\sum_{\mathbf{R}^{\prime} \neq \mathbf{0}} \mathbf{G}\left(\mathbf{0}, \mathbf{R}^{\prime}\right) \exp \left(i \mathbf{k}_{\mathbf{B}} \cdot \mathbf{R}^{\prime}\right) .
$$

Here we have used the equality $\mathbf{G}\left(\mathbf{R}_{i}, \mathbf{R}_{j}\right)=\mathbf{G}\left(\mathbf{0}, \mathbf{R}_{j}-\mathbf{R}_{i}\right)$ and $\mathbf{R}^{\prime}=\mathbf{R}_{j}-\mathbf{R}_{i}$. The dyadic $\boldsymbol{\beta}$ contains the geometrical information. If the dipoles are arranged in the $x-y$ plane, we can exploit the property of dipolar Green's function to divide the matrix [Eq. (2.6)] into two independent equations,

$$
\begin{gathered}
M_{z} p_{z}=E_{\text {inc }, z} \text { (Transverse), } \\
\mathbf{M}_{\|} \mathbf{p}_{\|}=\mathbf{E}_{\text {inc }, \|} \text { (In-plane), }
\end{gathered}
$$

where $M_{z}\left(\beta_{z}\right)$ is a scalar and $\mathbf{M}_{\|}\left(\beta_{\|}\right)$is a symmetric $2 \times 2$ matrix,

$$
\begin{gathered}
M_{z}=\frac{1}{\alpha}-\beta_{z}, \\
\mathbf{M}_{\|}=\frac{1}{\alpha} \mathbf{I}-\beta_{\|}=\left(\begin{array}{cc}
\frac{1}{\alpha}-\beta_{\|, 11} & -\beta_{\|, 12} \\
-\beta_{\|, 21} & \frac{1}{\alpha}-\beta_{\|, 22}
\end{array}\right) .
\end{gathered}
$$

For in-plane modes, the $p_{x}$ and $p_{y}$, the two components of $\mathbf{p}_{\|}$ along the $x$ and $y$ axes, respectively, are generally coupled to each other. We can diagonalize $\mathbf{M}_{\|}$with an orthogonal transformation $\mathbf{A}$,

$$
\mathbf{M}_{\|}^{\prime} \mathbf{p}_{\|}^{\prime}=\mathbf{E}_{\mathrm{inc}, \|}^{\prime},
$$

where

$$
\begin{gathered}
\mathbf{M}_{\|}^{\prime}=\mathbf{A} \mathbf{M}_{\|} \mathbf{A}^{\mathbf{T}}=\left(\begin{array}{cc}
M_{\|, 11}^{\prime} & 0 \\
0 & M_{\|, 22}^{\prime}
\end{array}\right), \\
\mathbf{p}_{\|}^{\prime}=\mathbf{A} \mathbf{p}_{\|}=\left(\begin{array}{l}
p_{\|, 1}^{\prime} \\
p_{\|, 2}^{\prime}
\end{array}\right), \\
\mathbf{E}_{\mathrm{inc}, \|}^{\prime}=\mathbf{A} \mathbf{E}_{\mathrm{inc}, \|}=\left(\begin{array}{l}
E_{\mathrm{inc}, 1}^{\prime} \\
E_{\mathrm{inc}, 2}^{\prime}
\end{array}\right) .
\end{gathered}
$$

The dipole polarizability of a sphere can be written as

$$
\alpha(\omega)=\frac{\varepsilon(\omega)-\varepsilon_{m}}{\varepsilon(\omega)+2 \varepsilon_{m}} a^{3},
$$

where $\varepsilon$ is the permittivity of the material of the nanosphere and $\varepsilon_{m}$ is the permittivity of the embedding medium. In this paper, we focus on the case in which the spheres are embedded in air where $\varepsilon_{m}=1$. After diagonalization, we can find a transverse in-plane mode (TI mode, dipole moment perpendicular to Bloch vector) and a longitudinal in-plane mode (LI mode, dipole moment parallel to Bloch vector), which are decoupled to each other. To take care of the radiation correction, we write 28 


$$
\frac{1}{\alpha} \rightarrow \frac{1}{\alpha}-i \frac{2 \omega^{3}}{3 c^{3}},
$$

such that

$$
\frac{1}{\alpha}=\left(1+\frac{3}{\varepsilon-1}\right) \frac{1}{a^{3}}-i \frac{2 \omega^{3}}{3 c^{3}} .
$$

The above radiation correction has been widely used in the literature (see, for example, Refs. 11, 12, 15, 23, and 24).

\section{QUASISTATIC LIMIT}

We first consider the quasistatic response for a 2D periodic array of lossless metal nanospheres. We use the Drude model to describe the permittivity as a function of frequency near the plasma resonance,

$$
\varepsilon(\omega)=1-\frac{\omega_{p}^{2}}{\omega(\omega+i v)},
$$

where $\omega_{p}$ is the plasma frequency and $v$ is the Drude damping term.
As all dipoles should be decaying in time, the normalmode frequency $\omega$ of the array should be complex with a negative imaginary part. However, because of the factor $e^{\mathrm{i} \omega \mathrm{r}_{i j} / c}$, the lattice sum $\boldsymbol{\beta}$ diverges when $\operatorname{Im}(\omega)<0$.

In the quasistatic limit, the speed of light is taken to be infinity $(c \rightarrow \infty)$. In addition, for lossless metal $(v=0)$, there is no energy loss and the polarizability has the simple form,

$$
\frac{1}{\alpha}=\frac{1}{a^{3}}\left(1-\frac{\omega^{2}}{\omega_{0}^{2}}\right),
$$

where $\omega_{0}=\omega_{p} / \sqrt{3}$ is the plasma resonance frequency for the sphere.

In the absence of radiation damping and current dissipation, the quality factor for each eigenmode (transverse or in plane) is infinity. That means the system has no coupling to the external environment. As a result, to investigate the eigenmodes of the system, we can simply set the incident wave $\mathbf{E}_{\text {inc }}$ to be zero. The solutions to dispersion [Eqs. (2.9) and (2.13)] are then $M_{z}=M_{11}^{\prime}=M_{22}^{\prime}=0$. The detailed form of dispersion relations for the eigenmodes of $2 \mathrm{D}$ periodic array of lossless metal nanospheres in quasistatic limit is then

$$
\left\{\begin{array}{l}
\omega^{2}=\omega_{0}^{2}\left(1-a^{3} \beta_{z}\right),(\text { Transverse }) \\
\omega^{2}=\omega_{0}^{2}\left\{1-\frac{a^{3}}{2}\left[\beta_{\|, 11}+\beta_{\|, 22}+\sqrt{\left(\beta_{\|, 11}-\beta_{\|, 22}\right)^{2}+4 \beta_{\|, 21}^{2}}\right]\right\}, \text { (Transverse in-plane) } \\
\omega^{2}=\omega_{0}^{2}\left\{1-\frac{a^{3}}{2}\left[\beta_{\|, 11}+\beta_{\|, 22}-\sqrt{\left(\beta_{\|, 11}-\beta_{\|, 22}\right)^{2}+4 \beta_{\|, 21}^{2}}\right]\right\} .(\text { Longitudinal in-plane) }
\end{array}\right.
$$

Here in quasistatic limit, the $\boldsymbol{\beta}$ is a dyadic of lattice sum of dipolar Green's function in which the retardation is disregarded,

$$
\begin{gathered}
\beta_{z}=-\sum_{\mathbf{R} \neq \mathbf{0}} \frac{1}{R^{3}} \exp \left(i \mathbf{k}_{\mathbf{B}} \cdot \mathbf{R}\right), \\
\boldsymbol{\beta}_{\|} \equiv\left(\begin{array}{cc}
\beta_{\|, 11} & \beta_{\|, 12} \\
\beta_{\|, 21} & \beta_{\|, 22}
\end{array}\right) \\
=\sum_{\mathbf{R} \neq \mathbf{0}} \frac{\exp \left(i \mathbf{k}_{\mathbf{B}} \cdot \mathbf{R}\right)}{R^{3}}\left(\begin{array}{cc}
3 R_{x}^{2} / R^{2}-1 & 3 R_{x} R_{y} / R^{2} \\
3 R_{x} R_{y} / R^{2} & 3 R_{y}^{2} / R^{2}-1
\end{array}\right) .
\end{gathered}
$$

As $\boldsymbol{\beta}$ can be expressed in $1 / d^{3}$ times a dimensionless number that is independent of $d$, we can conclude from Eq. (3.3) that the dispersion relations have bandwidths proportional to $a^{3} / d^{3}$ which corresponds to the coupling strength, same as the case for $1 \mathrm{D}$ chain. ${ }^{11}$ Because of such coupling coefficient, we can enhance the coupling effect among dipoles by increasing the radius and decreasing the lattice spacing.
However, we should also keep in mind that in order to guarantee the accuracy of the dipolar approximation, the radius $a$ must be small enough such that lattice spacing $d$ is not less than $3 a$; otherwise high-order Mie resonance beyond the dipolar resonance will emerge. ${ }^{29}$ To obtain results comparable with previous work, we set the radius of a sphere $a$ $=25 \mathrm{~nm}$ and the lattice spacing $d=75 \mathrm{~nm}$. The result for an infinite square lattice of silver spheres is plotted in Fig. 1.

From Fig. 1, we can see that at the $\Gamma$ point, the center of the first Brillouin zone (BZ), the two in-plane modes are degenerate at $\omega \approx 0.91 \omega_{0}$ which is close to that of the longitudinal mode for 1D chain, while the resonant frequency for transverse mode ( $\mathrm{T}$ mode) is about $1.15 \omega_{0}$ and higher than that for $1 \mathrm{D}$ chain. The behaviors of the three modes at different symmetric points can be explained by a simple restoring-force model. Within the quasistatic limit, the dipolar Green's function contains only the short-range interaction varying with distance in the form of $1 / R^{\prime 3}$; it is thus reasonable to consider the effect of only those nearest and secondnearest dipoles to the reference dipole. In order to understand qualitatively the dispersion relations at symmetric points of first BZ, we plot in Fig. 2 the relative phase of dipole mo- 


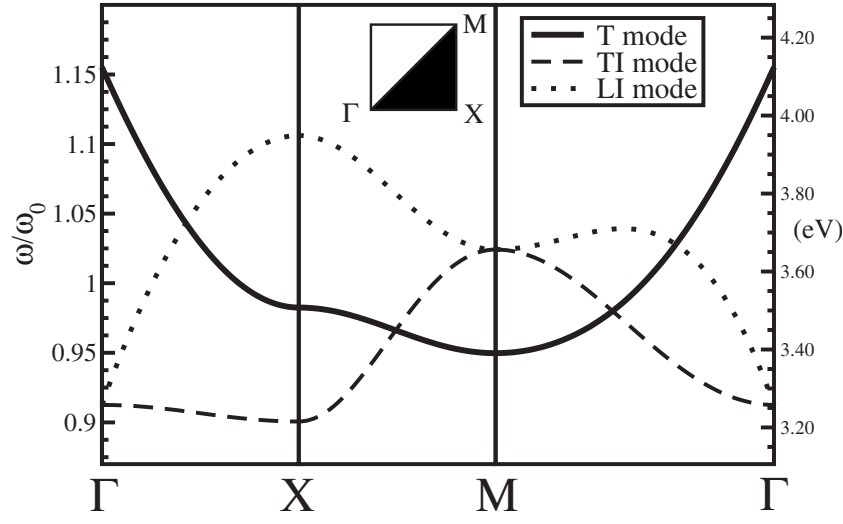

FIG. 1. Dispersion relations for the dipolar transverse mode (solid line), transverse in-plane mode (long dash line), and longitudinal in-plane mode (short dash line) in the quasistatic limit for an infinite square array of lossless $25 \mathrm{~nm}$ radii $\mathrm{Ag}$ spheres with a lattice constant of $75 \mathrm{~nm}$. The lattice sum is evaluated in a $2000 \times 2000$ grid. The $\omega_{0}$ for silver is $3.57 \mathrm{eV}$, and an absolute scale in $\mathrm{eV}$ is also presented in the right-hand side.

ments distributed near the reference particle for different symmetric points of first BZ of square lattice.

For a single particle, the plasmonic resonant frequency $\left(\omega_{0}\right)$ of the induced charges is provided by a restoring force that is due to the Coulomb attraction between the positive cores and the displaced negative electron charges. When there are many particles close together, the net surface charges on the other particles will provide additional Coulomb forces acting on the negative charge on the first particle, thus change the net restoring force. An increase (decrease) in restoring force will increase (decrease) the resonant frequency. To investigate these interaction forces, we summarize the orientations of the dipole moments for different symmetry points in Fig. 2.

For $\mathrm{T}$ mode, the dipole moments are perpendicular to the plane of the particle array. If the dipole moments of two neighboring particles are in phase (antiphase), the net surface charges on the other particle will add a force on the plasma of the first particle in a direction that is opposite to (the same as) the displacement of plasma, thus increase (decrease) the restoring force. At $\Gamma$ point, all dipoles are in phase. As a

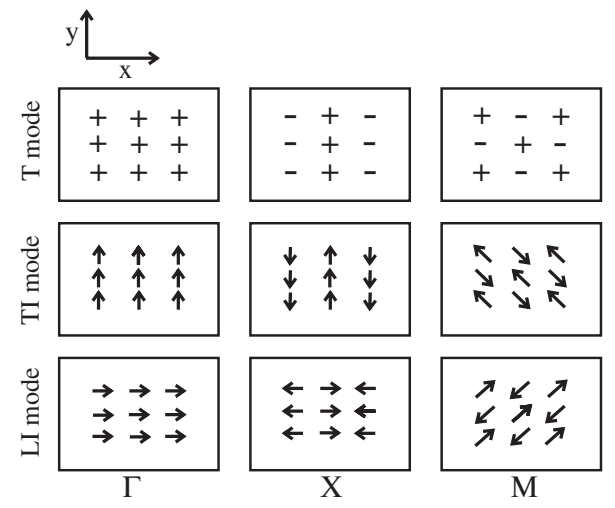

FIG. 2. The relative phase distributions of dipole moments for different symmetric points of first BZ near the reference particle that is at the center of the $3 \times 3$ array at $x-y$ plane. result, the resonant frequency $\omega_{\mathrm{T}}(\Gamma)$ is higher than $\omega_{0}$. At $X$ point, we can see that the electric fields from the four nearest dipoles cancel each other so that the forces provided by the four antiphase second-nearest dipoles become important. As a result, the resonant frequency $\omega_{\mathrm{T}}(X)$ is slightly lower than $\omega_{0}$. At $M$ point, the four nearest dipoles are all antiphase with the reference dipole. Therefore, the overall reduction in the restoring force is more than that for $X$ point, leading to a lower resonant frequency than that for $X$ point, namely, $\omega_{\mathrm{T}}(M)<\omega_{\mathrm{T}}(X)$.

For in-plane modes, the dipole moments are confined along the plane of particle array. The interaction can be classified into two kinds. We call the force from a parallel dipole that is positioned along (perpendicular to) the polar direction the longitudinal (transverse) interaction coupling. By inspecting the field generated by a dipole at a fixed short distance, one can easily see that the longitudinal coupling is larger than the transverse coupling. For the TI mode at $\Gamma$ point, two nearest particles provide longitudinal coupling that decrease the restoring force, while the other two nearest particles provide transverse coupling that increases the restoring force. Therefore, the final result is a reduction in restoring force, which results in a lower resonant frequency, namely, $\omega_{\mathrm{I}_{1}}(\Gamma)<\omega_{0}$. Since TI mode and LI mode have the same dipole arrangements (except a $90^{\circ}$ rotation) at $\Gamma$ point, they are degenerate and thus we have $\omega_{\mathrm{I}_{1}}(\Gamma)=\omega_{\mathrm{I}_{2}}(\Gamma)<\omega_{0}$. By similar analysis, one can see that the resonant frequency of the TI (LI) mode at the $X$ point is higher (lower) than that at the $\Gamma$ point. Therefore, we have $\omega_{\mathrm{I}_{1}}(X)<\omega_{0}$ and $\omega_{\mathrm{I}_{2}}(X)$ $>\omega_{0}$. The two in-plane modes are also degenerate at $M$ point and have a higher resonant frequency than $\omega_{0}$.

Due to symmetry, the slopes of the dispersions at zone center should be exactly zero. However, Fig. 2 shows that the group velocities $v_{g}$ at zone center appear to be finite near the zone center. To reveal the physics behind this strange behavior, a more detailed and careful inspection of functions near the zone center is required. This can be done by taking a straightforward differentiation of Eq. (3.3) at $\Gamma$ point. We will show that an integral method for dynamic case followed by taking the limit $c \rightarrow \infty$ in $k_{\omega}=\omega / c$ will be helpful for explaining the above issue. We will return to this issue in Sec. IV.

\section{DYNAMIC RESPONSE}

\section{A. Effective polarizability}

In this section, we will discuss the electrodynamic response of the 2D infinite array of metal nanospheres. We consider the retardation effect and the intrinsic loss of metal spheres. We solve this problem as a driven system in which the plasmonic spheres respond to an external driving field with a driving frequency that is a real number to investigate the steady state of a quasimode (resonant mode). As we stick with the real axis in frequency, the divergence problem we mentioned earlier for the quasistatic case will not occur. With an incident driving field and a finite Drude damping term, $v$, the extinction cross section of the whole system is nonzero. From Eqs. (2.9), (2.13), and (2.14), we have 


$$
\begin{gathered}
p_{z}=\alpha_{\mathrm{eff}, z} E_{\mathrm{inc}, z}, \\
p_{\|, 1}^{\prime}=\alpha_{\mathrm{eff}, 1} E_{\mathrm{inc}, 1}^{\prime}, \\
p_{\|, 2}^{\prime}=\alpha_{\mathrm{eff}, 2} E_{\mathrm{inc}, 2}^{\prime},
\end{gathered}
$$

where

$$
\begin{gathered}
\alpha_{\mathrm{eff}, z}=\frac{1}{M_{z}}, \\
\alpha_{\mathrm{eff}, 1}=\frac{1}{M_{\|, 11}^{\prime}}, \\
\alpha_{\mathrm{eff}, 2}=\frac{1}{M_{\|, 22}^{\prime}} .
\end{gathered}
$$

Here $\alpha_{\text {eff }}$ is defined as an effective polarizability that represents the effective response of a reference particle to the external incident field. This effective polarizability is contributed by not only the intrinsic polarizability of a metal nanosphere but also the scattering from other nanospheres located at periodic lattice sites. Hence, we have considered both single-particle properties and interparticle coupling in which geometrical information and periodic boundary condition are included. From the derivation of $\alpha_{\text {eff }}$ we note that in an infinite lattice (with one particle per unit cell) each particle has the same effective polarizability. Once the effective polarizability is known, the extinction cross section for each sphere is readily obtained from the optical theorem, ${ }^{30}$

$$
C_{\text {ext }}=\frac{4 \pi \omega}{c} \operatorname{Im}\left(\alpha_{\text {eff }}\right) .
$$

As a function of the driving frequency, the imaginary part of $\alpha_{\text {eff }}$ will exhibit peak(s), ${ }^{16}$ which is in good agreement with the dynamic dispersion relations obtained by finitechain solution. ${ }^{11}$ In addition, the mode-quality factors can also be obtained from the full width at half maximum (FWHM) of the peak. The advantage of this method is that it enables us to simultaneously see the dynamic dispersion relations and the quality factors for quasimodes.

We can easily obtain the resonant frequencies at a fixed spatial wave vector $k_{S}$ by seeking peak(s) of $\operatorname{Im}\left(\alpha_{\text {eff }}\right)$ as a function of $\omega$ as well as the dispersion relations by showing loci of resonant frequencies for varied $k_{S}$ in the first BZ. Second, the bandwidth of the resonant peak(s) of $\operatorname{Im}\left(\alpha_{\text {eff }}\right)$ can be related to the mode-quality factor by $Q=\omega_{r} / \Delta \omega$, where $Q$ is the quality factor, $\omega_{r}$ is the resonant angular frequency, and $\Delta \omega$ is the linewidth $(\sim$ FWHM $)$ of the quasimode. As a result, by plotting the map of $\operatorname{Im}\left(\alpha_{\text {eff }}\right)$ as a function of spatial wave vector $k_{S}$ and incident frequency $\omega$, we can observe both the dispersion relations and the mode qualities.

\section{B. Lattice sum of Green's function}

To obtain $\alpha_{\text {eff }}$, we have to evaluate the lattice sum $(S)$ of Green's function. In the dynamic dipolar Green's function, a term proportional to $e^{i \mathbf{k}_{\mathbf{B}} \cdot \mathbf{R}} e^{i \omega R / c} / R$ represents the long-range interaction, while the other terms represent the short-range $\left(\sim 1 / R^{3}\right)$ and intermediate-range $\left(\sim 1 / R^{2}\right)$ interactions. The lattice sums of short-range term and intermediate-range terms have good convergence and can be calculated numerically with a satisfactory accuracy, as long as the spatial lattice used in the calculation is large enough. However, the lattice sum of the long-range term does not converge for real $\omega$. In the literature, several methods have been developed to deal with such kind of lattice sum. A special method for summing the long-range term with the help of imaginary dipole array has been discussed by Belov et al. ${ }^{31}$ If the dipole array is dense, namely, the dipole spacing is far less than the incident wavelength, an integral method can be applied to evaluate that lattice sum. ${ }^{20,31}$

More rigorous and general methods are related to the application of Poisson's formula and the summation on reciprocal lattice. For summing the long-range term on an infinite and complete 2D lattice, the Ewald's method can be applied to split the term into two parts, with one fast converging on the spatial lattice and the other on reciprocal lattice. ${ }^{32}$ In our case, although the spatial lattice where the summation will be done is not complete because of the exclusion of origin, we can still rewrite the summation as a complete one accompanied by a self-radiated diverging term subtracted [see Eq. (A2)]. Actually a straightforward application of Ewald's method to the first complete series in Eq. (A2) is proven efficient in generating numerically accurate and quickly converging results, even when $z \rightarrow 0_{+}$. However, for summing such kind of long-range term on uncompleted lattice, Simovski et al. ${ }^{19}$ developed an interesting series which will be adopted below. The method is appealing mainly because it can help us to identify more clearly the mathematical contributions from different physical parameters.

While the form of the series in Ref. 19 was for rectangular lattice only, the idea is valid for arbitrary 2D periodic arrays with one particle in one unit cell. The original paper of Simovski et al. ${ }^{19}$ contains some typos, and for that reason, we rederived the fast-converging series following the work in Ref. 19 in Appendix A. Our result is more general and is valid for any $2 \mathrm{D}$ simple lattice with one particle per primitive cell. The result of the long-range lattice sum is

$$
\begin{aligned}
S\left(k_{\omega}, \mathbf{k}_{\mathbf{B}}\right) & \equiv \sum_{\mathbf{R} \neq \mathbf{0}} \frac{e^{i\left(k_{\omega} R+\mathbf{k}_{\mathbf{B}} \cdot \mathbf{R}\right)}}{R} \\
& =D+\frac{2 \pi i}{\Omega} \frac{1}{k_{z, 0}}-i k_{\omega}+\frac{2 \pi}{\Omega} \sum_{\mathbf{G} \neq \mathbf{0}}\left(\frac{1}{k_{z, \mathbf{G}}}-\frac{1}{G}\right),
\end{aligned}
$$

where $D$ is a constant for a specific lattice structure, G's are $2 \mathrm{D}$ reciprocal-lattice vectors, $\Omega$ is the area of one unit cell in real-space lattice, $k_{\omega}=\omega / c$ is wave number of incident wave, $k_{z, 0}=\sqrt{k_{\omega}^{2}-\left|\mathbf{k}_{\mathbf{B}}\right|^{2}}, \quad k_{z, \mathbf{G}}=\sqrt{\left|\mathbf{k}_{\mathbf{B}}+\mathbf{G}\right|^{2}-k_{\omega}^{2}}$, and $\operatorname{Im}\left(k_{z, 0}\right) \geq 0$ and $\operatorname{Im}\left(k_{z, \mathbf{G}}\right) \geq 0$.

Given a fixed $k_{\omega}$, as $\mathbf{k}_{\mathbf{B}}$ increases and crosses the light line, the second term $\left(2 \pi i / \Omega 1 / k_{z, 0}\right)$ in Eq. (4.8) experiences a divergence and goes from purely imaginary to purely 
real. It hence implies that the dispersions could also experience a singularity, which we will show below, at the light line.

The interesting quantity $D$ is found to possess definite physical meaning. As we can see from its definition (see Appendix A), D represents a geometrical effect in electrostatic limit as $k_{\omega}$ tends to zero, and it only depends on the lattice structure rather than any wave nature of the summation. On the other hand, both the second term and third term in Eq. (4.8) depend on the wave number $k_{\omega}$ and wave vector $\mathbf{k}_{\mathbf{B}}$ so that they mainly represent the wave nature of the summation. Last, the series of correction terms is evaluated over reciprocal lattice excluding origin so that it depends on both the geometrical effect and the wave nature. However, the correction series is much smaller than other terms. As a result, the lattice sum in Eq. (4.8) is dominated by the first three terms and is very fast converging.

When we evaluate $D$, we can replace the series in Eq. (A8) with a two-dimensional integral in the reciprocal space,

$$
\Omega_{G} \sum_{G}^{\prime} \rightarrow \int_{0}^{2 \pi} d \theta \int_{g_{\min }}^{\infty} g d g
$$

where $\Omega_{G}=4 \pi^{2} / \Omega$ is the area of unit cell of reciprocal lattice and we have assumed that the replacement is rigorous as long as the integral is taken outside a finite circle centered at origin and with radius $g_{\min }$. Therefore,

$$
\begin{aligned}
D & =\lim _{z \rightarrow 0_{+}}\left(\frac{\Omega_{G}}{2 \pi} \sum_{G}{ }^{\prime} \frac{e^{-z G}}{G}-\frac{1}{z}\right) \\
& =\lim _{z \rightarrow 0_{+}}\left(\frac{1}{2 \pi} \int_{0}^{2 \pi} d \theta \int_{g_{\min }}^{\infty} g d g \frac{e^{-z g}}{g}-\frac{1}{z}\right) \\
& =\lim _{z \rightarrow 0_{+}}\left(\int_{g_{\min }}^{\infty} e^{-z g} d g-\frac{1}{z}\right)=\lim _{z \rightarrow 0_{+}}\left(\frac{e^{-z g_{\min }}}{z}-\frac{1}{z}\right)=-g_{\min } .
\end{aligned}
$$

In Eq. (4.10), we have shown that $D$ must be a negative real number with a magnitude $g_{\text {min }}$ which is the radius of a finite circle, and we have assumed that the infinite integral taken out of that circle is exactly equivalent to the infinite lattice sum in reciprocal space. We introduce a parameter $U$ sufficiently large and let the summation in Eq. (4.10) be numerically calculated in the region $0<G \leq U / z$. We then define a quantity

$$
L \equiv \frac{\Omega_{G}}{2 \pi} \sum_{0<G \leq U / z} \frac{e^{-z G}}{G} \rightarrow L=\int_{g_{\min }}^{z \rightarrow 0_{+}} e^{-z g} d g=\frac{e^{-z g_{\min }}-e^{-U}}{z}
$$

Here we again employ our assumption that an infinite lattice summation can be rigorously replaced by a $2 \mathrm{D}$ integral with $g_{\min }$ as inner bound and infinity as outer bound. Hence we have two methods of evaluating $D$ by approaching to the zero-point limit of two different functions,

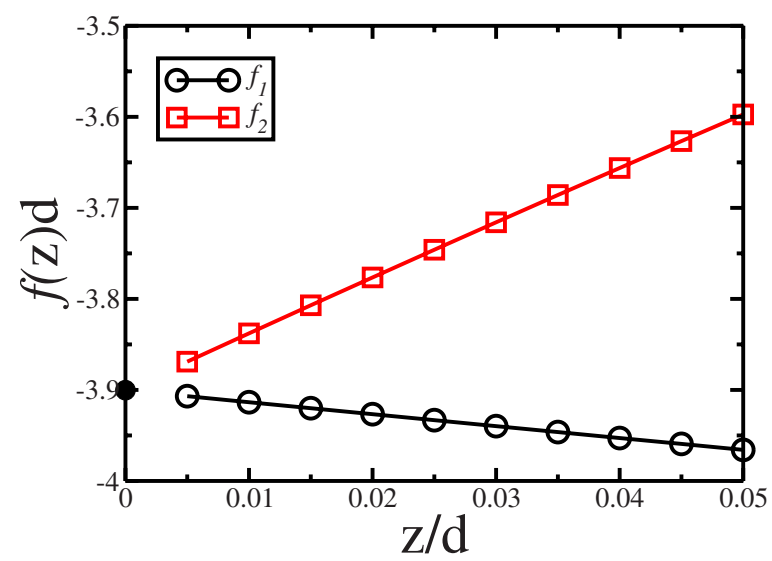

FIG. 3. (Color online) Plots of $f_{1}$ (open circle) and $f_{2}$ (open square) at small $z$ region. The values of $D$, marked by the solid circle at $z=0$ axis and extrapolated out of $f_{1}$ and $f_{2}$, respectively, coincident with each other with a difference of order $10^{-4}$. The $U$ is set to be 10 here.

$$
D=-g_{\min }=\lim _{z \rightarrow 0_{+}} f_{1}(z)=\lim _{z \rightarrow 0_{+}} \frac{\ln \left(z L+e^{-U}\right)}{z}
$$

and

$$
D=\lim _{z \rightarrow 0_{+}} f_{2}(z)=\lim _{z \rightarrow 0_{+}}\left(L+\frac{e^{-U}-1}{z}\right) .
$$

In Fig. 3 we plot the $f_{1}(z)$ and $f_{2}(z)$ for square lattice in small $z$ region and find excellent linear convergence for the two functions. Extrapolations at $z=0$ are done for the two lines and the results coincide with each other to the order of $10^{-4}$. Then we take the averaged number of the two extrapolated results to be final result of $D$. For square lattice $D=$ $-3.9002 / d$, in good agreement with the result obtained by Simovski et al. ${ }^{33}$; with a similar approach, we obtain $D=$ $-4.2133 / d$ for triangular lattice. The parameter $U$ can of course be increased to obtain arbitrary precision, but the current results are good enough for our purpose.

\section{Results and discussion}

For the three modes propagating on an infinite 2D nanosphere square lattice, we plot in Figs. 4(a)-4(c) the dimensionless $\operatorname{Im}\left(\alpha_{\text {eff }}\right) / a^{3}$ in a color map as a function of the incident frequency $\omega$ and wave vector $\mathbf{k}_{\mathbf{B}}$ in $\Gamma X$ direction. In Fig. 4(d), we also plot $\operatorname{Im}\left(\alpha_{\text {eff }}\right) / a^{3}$ versus $\omega$ for the three modes at fixed values of $k$ inside $(k d=0.19)$ and outside $(k d=2.50)$ the light cone $(k<\omega / c)$ in order to visualize the widths of the peaks of $\operatorname{Im}\left(\alpha_{\text {eff }}\right)$ and to make the comparison of mode quality easier. The short-range and intermediate-range terms in dipolar Green's function are summed over 3000 $\times 3000$ grids, while the long-range terms are evaluated by Eq. (4.8), with the correction terms summed in the region $|\mathbf{G}| \leq 320 \pi / d$. In the numerical calculation we use a Drude model fitted from experimental data for silver, ${ }^{34,35}$ 

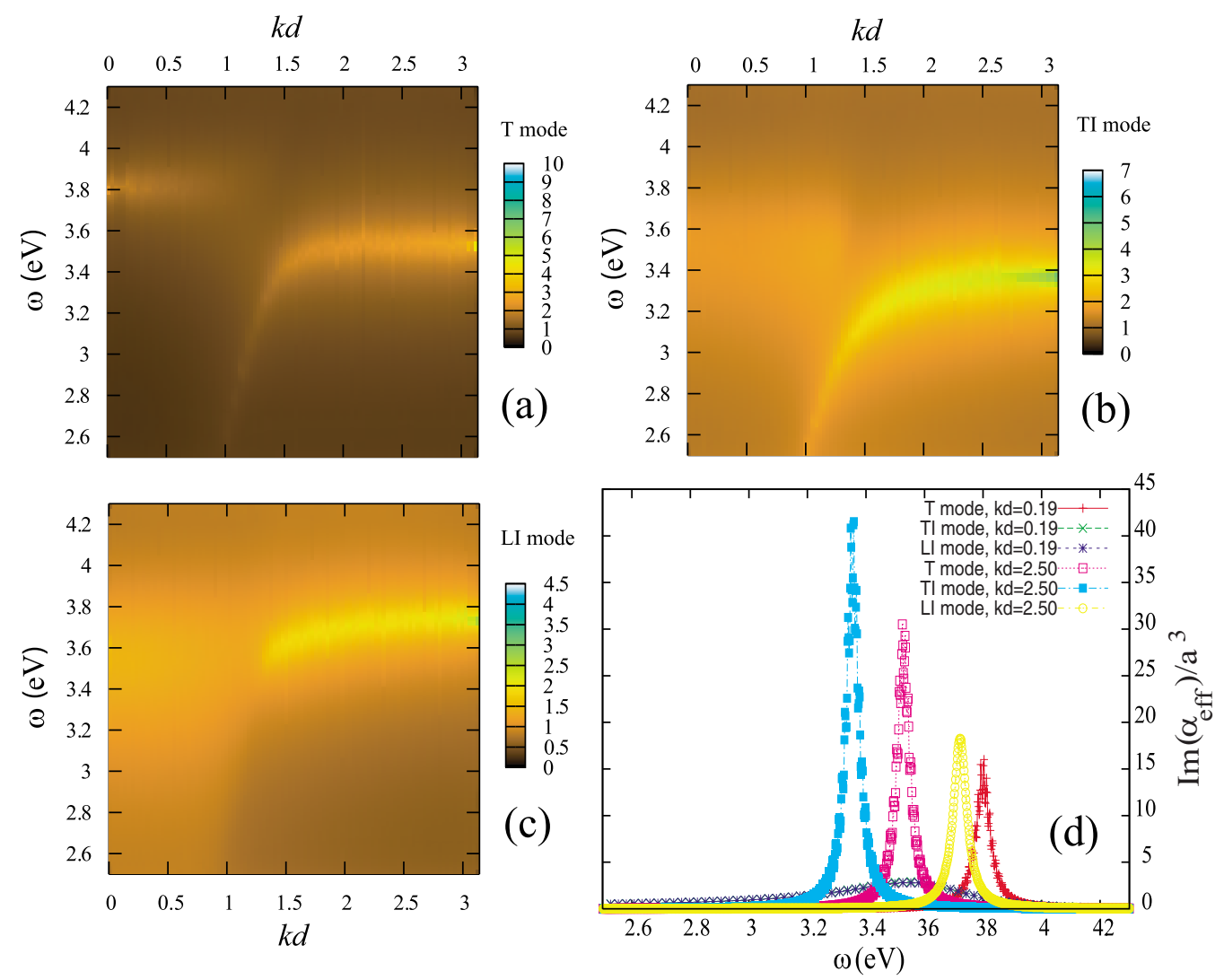

FIG. 4. (Color online) Dispersion diagrams for the quasimodes of silver nanospheres in square array along $\Gamma$-X section of the first BZ. The radii of spheres are $25 \mathrm{~nm}$, with center-to-center distance of $75 \mathrm{~nm}$. (a) T mode. (b) TI mode. (c) LI mode. The color bars in panels (a)-(c) show the relative values of $\operatorname{Im}\left(\alpha_{\text {eff }}\right)$, but the values and colors are rescaled to give clearer visualization. (d) $\operatorname{Im}\left(\alpha_{\text {eff }}\right) / a^{3}$ versus $\omega$ for the three modes at fixed values of $k$ inside $(k d=0.19)$ and outside $(k d=2.50)$ the light cone. Note that the curves for TI and LI modes at $k d=0.19$ almost coincide because they are close to the degeneracy point at $k=0$.

$$
\varepsilon(\omega)=\varepsilon_{a}-\frac{\left(\varepsilon_{b}-\varepsilon_{a}\right) \omega_{\mathrm{pl}}^{2}}{\omega(\omega+i v)},
$$

with parameters $\varepsilon_{a}=5.45, \varepsilon_{b}=6.18, \omega_{\mathrm{pl}}=11.34 \mathrm{eV}$ (singlesphere resonant frequency $\omega_{0}=3.57 \mathrm{eV}$ ), and $v=0.05 \mathrm{eV}$ to obtain results for silver nanospheres. All the spheres have the same radius of $25 \mathrm{~nm}$ and a center-to-center spacing of 75 $\mathrm{nm}$, the same as our quasistatic calculation and some previous work by others.

From Fig. 4, we can observe the following phenomena of the three modes: (1) inside the light-cone, the $\mathrm{T}$ mode is rather well defined near the zone-center, while the two inplane modes are fuzzy; (2) the bands of T mode and TI mode drop suddenly and significantly when they meet the light line, while for LI mode the band drops down continuously with a small negative $v_{g}$ before it meets light line; and (3) outside the light-cone, all three modes are well defined, with relatively high quality factors.

These observations can be explained as follows. First, all three modes have radiation damping within the light cone. For a single dipole, the far-field time-averaged Poynting vector $\overline{\boldsymbol{\rho}}$ radiated by this dipole has an angular distribution in the form $\bar{\rho} \propto \sin ^{2} \theta,{ }^{27}$ where $\theta$ is the angle of Poynting vector measured from the dipole axis (see Fig. 5). For T mode (inplane modes), the energy radiated within (out of) the plane is strong. Therefore, inside the light cone the two in-plane modes are very leaky, while the $\mathrm{T}$ mode has relatively good quality because most of the energy is kept in the plane. Second, the sharp drop from the zone center toward the light line has been observed for the T mode of $1 \mathrm{D}$ chains ${ }^{8,11,15,16}$ and is the result of the strong coupling of the plasmonic mode with free photons in vacuum. We see the same phenomena here in the $2 \mathrm{D}$ cases. The $\mathrm{T}$ mode and TI mode in $2 \mathrm{D}$ arrays are developed from the two degenerate transverse modes of an infinite 1D chain, while the other in-plane LI mode originates from the longitudinal mode of the $1 \mathrm{D}$ chain. That is why we call the in-plane modes "TI/LI mode." The sudden dips in dispersions for both $\mathrm{T}$ mode and TI mode indicate a strong coupling with free photons. With dipole moments orthogonal to the propagating wave vector (and hence parallel

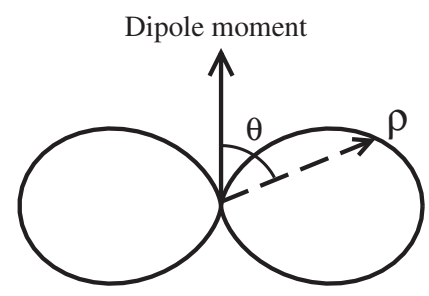

FIG. 5. The angular distribution of Poynting vector of the field radiated by a single electric dipole. The figure defines the angle $\theta$. 
to the $E$ field), these two modes can be directly excited by free photons. In contrast, the LI mode has no such coupling with free photons as the dipole moment is parallel to wave vector. In $1 \mathrm{D}$ silver-sphere chain, the longitudinal mode joints smoothly from inside the light cone to the guided modes outside the light cone. However, the LI mode of 2D silver-sphere array shows a pseudocoupling with light line at which the band's slope $\left(v_{g}\right)$ shows a negative-to-positive transition. We will show below that the $v_{g}$ inside the light cone for the LI mode of this specific 2D array is inherently negative, and thus the LI mode has to bend downward in frequency before it meets the light line and has to turn upward when it becomes a guided mode outside the light cone. Third, outside the light cone, the phase velocities of all three modes are larger than light speed $c$, the field must have an exponential decay in the direction perpendicular to the plane, and no energy can be radiated. Thus, the guided mode qualities for all three modes are all determined by absorption loss, which is dictated by the $v$ in the Drude model.

As the imaginary part of $M$ is small, the peak of $\operatorname{Im}\left(\alpha_{\mathrm{eff}}\right)$ coincides with the contour $\operatorname{Re}(M)=0$. As we discussed before, the geometrical constant $D$ extracted out of the lattice sum of long-range interaction is included in $\operatorname{Re}(M)$; therefore it will affect the resonant frequencies. For example, the magnitude of $D$ in triangular lattice is larger than that in square lattice, resulting in a small upshift of the resonant frequency for transverse mode and a downshift for in-plane modes in Brillouin-zone center. The larger magnitude of $D$ corresponds to a more close-packed real-space lattice, and thus the resonant frequency(ies) of transverse (in-plane) mode(s) will be higher (lower) in a more close-packed lattice when all the particles are in phase.

For the square lattice, the dispersion along $\Gamma M$ shows similar behavior as in the $\Gamma X$ direction: Inside the light cone the transverse mode has good qualities, while the two inplane modes are hardly observable; the T mode and TI mode are strongly coupled with free photons, while the LI mode has a pseudocoupling to free photons; last, outside the light cone, the guided qualities are determined by the absorption loss. We extract the peak positions of $\operatorname{Im}\left(\alpha_{\text {eff }}\right)$ for the three modes along three symmetric directions in the first BZ and plot them in Fig. 6 . We choose to display the loci of $\operatorname{Im}\left(\alpha_{\text {eff }}\right)$ only in Fig. 6, as the figure will be too busy if we display the information like Fig. 4.

We can see that the dispersions inside the light cone [as defined by peaks of $\left.\operatorname{Im}\left(\alpha_{\text {eff }}\right)\right]$ are almost isotropic, so that the frequencies only depend on the magnitude of the wave vector $\mathbf{k}_{\mathbf{B}}$. All the three modes have very flat bands inside the light cone, indicating very small $v_{g}$. To understand the underlying physics, it is helpful to seek approximate close-form solutions. As the sphere spacing of $75 \mathrm{~nm}$ is much smaller than the interested wavelengths, which is near or larger than the single-sphere resonant wavelength of $348 \mathrm{~nm}$, the whole 2D dipole array can be treated as a dense array so that the lattice sum can be approximated by an integral, and we can therefore obtain full-analytical expressions of the lattice sum of Green's function. ${ }^{20}$ We derive the integral form of the lattice sums of dipolar Green's function in Eq. (2.8) for the three modes following the work in Ref. 20 (see Appendix B). The results are

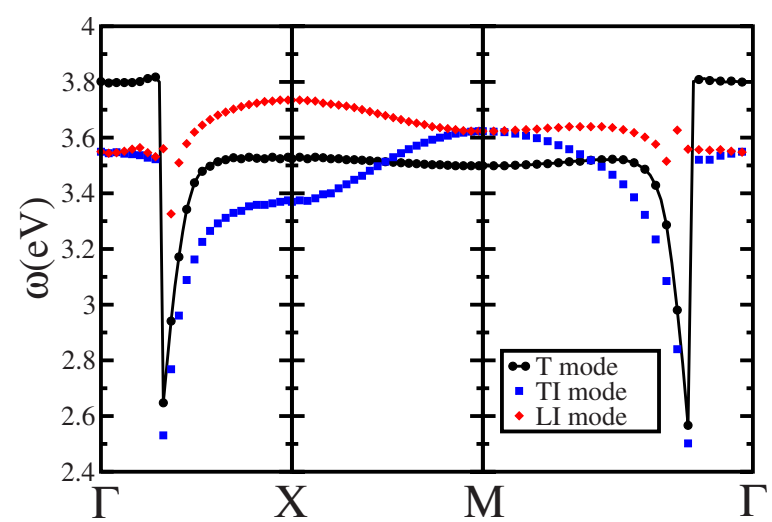

FIG. 6. (Color online) Dispersion relations for the three plasmonic modes in first $\mathrm{BZ}$ for $\mathrm{Ag}$ nanospheres arranged in a square lattice. The radii of spheres are $25 \mathrm{~nm}$, with lattice constant of 75 $\mathrm{nm}$. Line with circles represents the transverse mode ( $\mathrm{T}$ mode), squares represent the transverse in-plane mode (TI mode), and diamonds the longitudinal in-plane mode (LI mode).

$$
\begin{gathered}
\beta_{\mathrm{T}}=\frac{2 \pi}{\Omega}\left(I_{2}-I_{1}\right) \quad(\text { Transverse mode }), \\
\beta_{\mathrm{TI}}=\frac{2 \pi}{\Omega}\left(I_{2}+I_{3}\right) \quad(\text { Transverse in-plane mode }), \\
\beta_{\mathrm{LI}}=\frac{2 \pi}{\Omega}\left(I_{1}-I_{3}\right) \quad \text { (Longitudinal in-plane mode) }
\end{gathered}
$$

where the $I_{1}, I_{2}$, and $I_{3}$ are integrals that are slow varying with scalar wave number $k_{B}$ (see Appendix B).

With these results, we can now explain the apparent finite slope for quasistatic dispersion near the zone center in Sec. III. Let us start from a dynamic situation with a finite $k_{\omega}$ $=\omega / c$, and we replace the $2 \mathrm{D}$ lattice sums with the integrals whose details are shown in Appendix B. The integral methods should be a good approximation near the zone center because the wavelength is much greater than the lattice spacing. Therefore Eq. (3.3) can be rewritten as $\omega_{\sigma}^{2}=\omega_{0}^{2}(1$ $-a^{3} \beta_{\sigma}$ ), where $\sigma$ denotes the mode type and $\beta_{\sigma}$ is just the same as in Eqs. (4.15)-(4.17). Next, we differentiate $I_{\sigma}$ with respect to $k_{B}$ and then take the limit $k_{\omega}$ to zero (quasistatic), making the derivatives near zone center for $I_{2}$ and $I_{3}$ approach to zero and $O\left(k_{B}\right)$, respectively. For the $I_{1}$, the result will be determined by the competition between $k_{\omega}$ and $k_{B}$. Given that the quasistatic solutions only have physical meaning when $k_{\omega} \rightarrow 0$, if $k_{\omega} \gg k_{B}$, which means $k_{B}=0$, the $v_{g}$ will be exactly zero at $\Gamma$ point; otherwise, $k_{B} \gg k_{\omega} \rightarrow 0$, then we have

$$
\left.\frac{\partial I_{1}}{\partial k_{B}}\right|_{k_{\omega} \rightarrow 0}=-1+O\left(k_{B}\right) .
$$



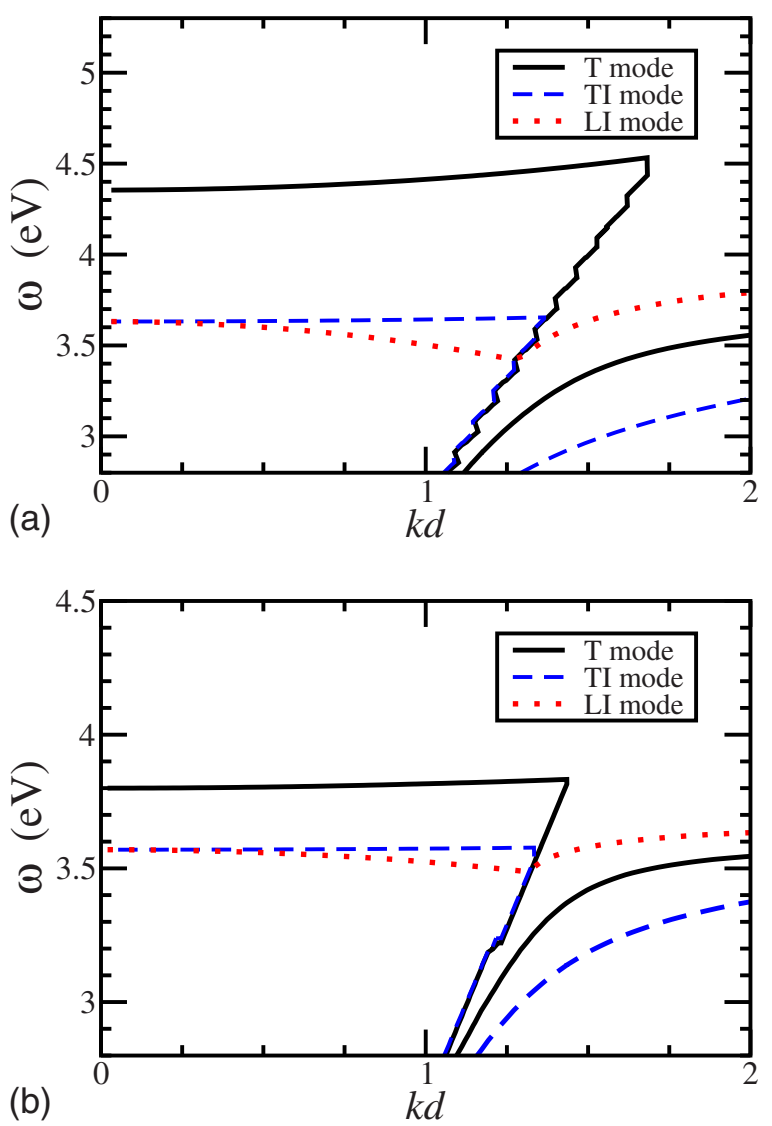

FIG. 7. (Color online) $\operatorname{Re}(M)=0$ contours for the transverse mode (solid line), transverse in-plane mode (long dash line), and longitudinal in-plane mode (short dash line) in the first Brillouin zone for Ag nanospheres in a square lattice. The results are computed with the integral approximation. The radii of spheres are 25 $\mathrm{nm}$, with center-to-center distance $75 \mathrm{~nm}$. The two results correspond to two Drude Models (a) using Eq. (3.1) and (b) using Eq. (4.14). $v=0.05 \mathrm{eV}$.

Thus from Eqs. (4.15)-(4.17) the $v_{g}$ of different modes near zone center shown in Fig. 1 can be explained. All modes must have $v_{g}$ exactly zero at $\Gamma$ point. In the vicinity of the zone center, the dispersion for TI mode is parabolic, while the slopes for T mode and LI mode have opposite signs and finite magnitudes.

Next, we continue to discuss the dispersion behavior for electrodynamic cases. We now have full-analytical form of $\operatorname{Im}\left(\alpha_{\text {eff }}\right)$ as $\operatorname{Im}\left(\alpha_{\text {eff, } \sigma}\right)=-\operatorname{Im}\left(M_{\sigma}\right) /\left|M_{\sigma}\right|^{2}$. Moreover we must stress again that because the imaginary part of $M$ is much smaller than the real part, the peak of $\operatorname{Im}\left(\alpha_{\text {eff }}\right)$ essentially coincides with the contour $\operatorname{Re}(M)=0$. So, we plot the $\operatorname{Re}(M)=0$ contours for the three modes in Fig. 7, where $M$ can be analytically evaluated using the integral method. Figures 7(a) and 7(b) correspond to the Drude model of Eqs. (3.1) and (4.14), respectively. The similarity of the results shown in Figs. 7(a) and 7(b) shows that the salient features of the dispersion do not depend on the details of the material parameters. From Appendix B, we know that only $I_{2}$ has $i / \sqrt{k_{\omega}^{2}-k_{B}^{2}}$ divergence. Actually, $2 \pi I_{2} / \Omega$ is just the integral form of $k_{\omega}^{2} S$, which stands for the transverse long-range dipole interaction. From Eqs. (4.15)-(4.17), we note that $I_{2}$ exists for T and TI but not for LI. So, we see that it is the transverse long-range dipole interaction that accounts for the light-line coupling. With the same Drude model, the dispersion found by integral method in Fig. 7(b) is in very good agreement with the dispersion relations shown in Fig. 6, particularly for small $k_{B}$, where the wavelength of the Bloch wave is large and the phase distribution of dipole moments is quite slow varying. Moreover, the small pseudocoupling between LI mode and light line is now clearly seen. In addition, the flat bands near the center of Brillouin zone are reproduced by the integral methods.

For estimating $v_{g}$, the simple form of the Drude model as in Eq. (3.1) is employed in the following discussion. For an implicit dispersion relation $f\left(\omega, k_{B}\right)=0$, where $f=\operatorname{Re}(M)$, the $v_{g}$ is given by

$$
\frac{d \omega}{d k_{B}}=-\frac{\partial f / \partial k_{B}}{\partial f / \partial \omega}
$$

Noting that

$$
\begin{gathered}
\frac{\partial \operatorname{Re}\left(M_{\sigma}\right)}{\partial \omega} \approx \frac{\partial \operatorname{Re}\left(\frac{1}{\alpha}\right)}{\partial \omega}=-\frac{2 \omega}{a^{3} \omega_{0}^{2}}, \\
\frac{\partial \operatorname{Re}\left(M_{\sigma}\right)}{\partial k_{B}}=-\frac{\partial \operatorname{Re}\left(\beta_{\sigma}\right)}{\partial k_{B}},
\end{gathered}
$$

we put Eqs. (2.19), (3.1), (4.20), and (4.21) into Eq. (4.19) and have

$$
\begin{aligned}
\frac{d \omega_{\sigma}}{d k_{B}} & =-\frac{\partial \operatorname{Re}\left(M_{\sigma}\right)}{\partial k_{B}} / \frac{\partial \operatorname{Re}\left(M_{\sigma}\right)}{\partial \omega} \\
& \approx-\frac{a^{3} \omega_{0}^{2}}{2 \omega_{\sigma}} \frac{\partial \operatorname{Re}\left[\beta_{\sigma}\right]}{\partial k_{B}} \equiv \frac{\gamma_{\sigma} k_{B} \omega_{0}^{2}}{\omega_{\sigma}},
\end{aligned}
$$

where the parameter $\gamma_{\sigma}$ depends on the mode $\sigma$. For the T mode, we have (see Appendix $\mathrm{B}$, and in the following $R$ denotes $R_{\min }$ for short)

$$
\begin{aligned}
\operatorname{Re}\left[\beta_{\mathrm{T}}\left(k_{\omega}, k_{B}\right)\right] \approx & \frac{2 \pi}{\Omega}\left\{\frac{3\left[\cos \left(k_{\omega} R\right)-\sin \left(k_{\omega} R\right)\right]}{4 R}\left(k_{B} R\right)^{2}\right. \\
& \left.-\frac{\cos \left(k_{\omega} R\right)+k_{\omega} R \sin \left(k_{\omega} R\right)}{R}\right\} \\
\gamma_{\mathrm{T}}= & 12\left[\sin \left(k_{\omega} R\right)-\cos \left(k_{\omega} R\right)\right] \gamma_{0}
\end{aligned}
$$

for the TI mode,

$$
\begin{aligned}
& \operatorname{Re}\left[\beta_{\mathrm{TI}}\left(k_{\omega}, k_{B}\right)\right] \approx \frac{2 \pi}{\Omega}\left\{\frac{7 \cos \left(k_{\omega} R\right)-5 \sin \left(k_{\omega} R\right)}{16 R}\left(k_{B} R\right)^{2}\right. \\
&\left.+\frac{\cos \left(k_{\omega} R\right)-k_{\omega} R \sin \left(k_{\omega} R\right)}{2 R}\right\}, \\
& \gamma_{\mathrm{TI}}=\left[5 \sin \left(k_{\omega} R\right)-7 \cos \left(k_{\omega} R\right)\right] \gamma_{0},
\end{aligned}
$$

for the LI mode, 


$$
\begin{aligned}
& \operatorname{Re}\left[\beta_{\mathrm{LI}}\left(k_{\omega}, k_{B}\right)\right] \approx \frac{2 \pi}{\Omega}\left[\frac{-3 \cos \left(k_{\omega} R\right)+9 \sin \left(k_{\omega} R\right)}{16 R}\left(k_{B} R\right)^{2}\right. \\
&\left.+\frac{\cos \left(k_{\omega} R\right)-k_{\omega} R \sin \left(k_{\omega} R\right)}{2 R}\right] \\
& \gamma_{\mathrm{LI}}=\left[-9 \sin \left(k_{\omega} R\right)+3 \cos \left(k_{\omega} R\right)\right] \gamma_{0}
\end{aligned}
$$

where $\gamma_{0}=\pi a^{3} R / 8 \Omega$.

Thus the dispersion relations for small wave vector in the light cone are approximately

$$
\omega_{\sigma}^{2}\left(k_{B}\right) \approx \omega_{\sigma}^{2}(0)+\gamma_{\sigma} \omega_{0}^{2} k_{B}^{2}
$$

Note that for $a / d=1 / 3, R=d / 1.438$, and $\Omega=d^{2}$ for square lattice, the magnitude of $\gamma_{0} / d^{2}$ is as small as 0.01 ; moreover, by putting $k_{\omega} R \simeq 1$ (for silver nanospheres in square lattice) into Eqs. (4.24), (4.26), and (4.28), we obtain $\gamma_{\mathrm{T}} \simeq 3.61 \gamma_{0}$, $\gamma_{\mathrm{TI}} \simeq 0.43 \gamma_{0}$, and $\gamma_{\mathrm{LI}} \simeq-5.95 \gamma_{0}$. As a result, the dispersion relations are very flat for small $k_{B}$ in the light cone, with the magnitudes of $v_{g}$,

$$
\begin{aligned}
\left|v_{g, \sigma}\right| & =\left|\frac{d \omega_{\sigma}}{d k_{B}}\right| \approx\left|\frac{\gamma_{\sigma} k_{B} \omega_{0}^{2}}{\omega_{\sigma}}\right| \\
& \approx\left|\frac{\gamma_{0}}{d^{2}}\left(k_{B} d\right)\left(\frac{\omega_{0} d}{c}\right) c\right| \sim\left|\left(k_{B} d\right) 0.01 c\right| .
\end{aligned}
$$

In addition, in the light cone the dispersion relations as given by Eq. (4.29) agree quite well with those obtained by $\operatorname{Re}(M)=0$ contours (see Fig. 7).

With the help of integral method, we have obtained approximate analytical expressions of the dispersion relations for small wave vectors. In contrast to the propagating plasmonic modes of $1 \mathrm{D}$ chain, $8,11,15,16$ the dispersion relations for the quasimodes of $2 \mathrm{D}$ array are much more slow varying with $k_{B}$ in the light cone. Mathematically, the "flat band" phenomena are associated with the slow-varying special functions $J_{0}, J_{1}$ in small $k_{B}$ regime. Also, the pseudolight-line coupling for LI mode can now be understood more. Although the down-and-up feature in LI dispersion looks similar to that for coupling with free photons, the mechanisms behind are different: once the dispersions reach the light line, the interparticle coupling coefficient of $\mathrm{T}$ and TI modes diverges, indicating a strong renormalization, while the LI mode comes to a minimal resonant frequency, which is required by the negative $\gamma_{\mathrm{LI}}$ in Eq. (4.28) coming from some special functions particular for $2 \mathrm{D}$ systems. Although $\gamma_{\mathrm{LI}}$ is related to lattice structure and resonant frequency through $k_{\omega} R$, within a quite broad range of $k_{\omega} R$ the $\gamma_{\mathrm{LI}}$ is kept negative, hence the negative $v_{g}$ and pseudocoupling with light line will also be seen in 2D systems other than square silversphere arrays, as long as the integral method is still valid. Also, within this method any lattice-dependent anisotropy is eliminated by the special functions for $2 \mathrm{D}$ systems and a small $\gamma_{0} / d^{2}$ is always reached, hence isotropic and flat bands inside light cone also exist in other 2D dense array with different structures or materials. Due to the high quality and nearly zero $v_{g}$ for transverse mode, 2D metal nanosphere array is a good candidate for optical storage, optical cavity, subwavelength imaging ${ }^{9}$ near the resonant frequency.

\section{CONCLUSION}

In summary, we have investigated the plasmonic modes on 2D periodic arrays. The particle arrays are treated in coupled-dipole approximation. We solved the problem first in the quasistatic limit and then we present the dynamical results. In the dynamic solution, the dispersion relations corresponding to quasimodes are obtained through an eigendecomposition method, which gives an effective polarizability describing the collective response of an infinite system to external driving monochromatic wave that is periodic in the $x-y$ plane. The dispersion relations and quality factors are simultaneously related to the imaginary part of the effective polarizability, which is affected separately by the singleparticle properties and interparticle coupling presented by the lattice sum of dipolar Green's functions. This method allows for the discussion of leaky modes inside the light cone.

For the quasistatic case, the relative positions of eigenfrequencies of three eigenmodes, particularly at symmetric points in the first BZ, can be explained by a simple restoringforce model. For dynamic case, inside the light cone, transverse mode has a relatively high mode quality, while the two in-plane modes show stronger radiation damping. Similar to the case of 1D chain, near the light line, direct coupling with free photons leads to a significant renormalization of the transverse mode and transverse in-plane mode. Moreover, interparticle coupling in a 2D system leads to negative group velocity for longitudinal in-plane mode inside the light cone. The mode qualities outside the light cone are dominated by the absorption loss of single particle. More interestingly, we demonstrate that the isotropic and flat bands observed inside the light cone can be qualitatively explained by an integral method which gives analytic expressions of dispersion relations. For 2D metal-sphere periodic arrays, we trace the small group velocities of the leaky modes within light cone to an intrinsic property of a dense array.

\section{ACKNOWLEDGMENTS}

We thank Jack Ng, Huanyang Chen, and Jun Mei for helpful discussions and Ting Li for her kindly help on the preparation of Fig. 2. This work is supported by a Hong Kong Central Allocation under Grant No. HKUST3/06C. Computation resources are supported by Shun Hing Education and Charity Fund.

\section{APPENDIX A}

Let

$$
Q\left(\mathbf{r}, k_{\omega}, \mathbf{k}_{\mathbf{B}}\right) \equiv \sum_{\mathbf{R}} e^{i \mathbf{k}_{\mathbf{B}} \cdot \mathbf{R}} \frac{e^{i k_{\omega}|\mathbf{r}-\mathbf{R}|}}{|\mathbf{r}-\mathbf{R}|},
$$

where $\mathbf{R}(\mathbf{r})$ denote the displacement vectors of real-lattice sites (field point), $k_{\omega}=\omega / c$, and $\mathbf{k}_{\mathbf{B}}$ is the Bloch wave vector. The lattice sum is related to the limit of above series by 


$$
S\left(k_{\omega}, \mathbf{k}_{\mathbf{B}}\right) \equiv \sum_{\mathbf{R}}{ }^{\prime} e^{i \mathbf{k}_{\mathbf{B}} \cdot \mathbf{R}} \frac{e^{i k_{\omega} R}}{R}=\lim _{r \rightarrow 0} Q\left(\mathbf{r}, k_{\omega}, \mathbf{k}_{\mathbf{B}}\right)-\lim _{z \rightarrow 0_{+}} \frac{e^{i k_{\omega} \sigma^{z}}}{z},
$$

where $\Sigma^{\prime}$ means a summation excluding the origin and the $\lim _{z \rightarrow 0_{+}}\left(e^{i k_{\omega} z} / z\right)$ is the self-radiated field of the reference particle at the origin.

When the summation over real lattice is to be done on $2 \mathrm{D}$ array, we know from Poisson's summation formula ${ }^{32}$ that

$$
Q\left(\mathbf{r}, k_{\omega}, \mathbf{k}_{\mathbf{B}}\right)=\sum_{\mathbf{G}} \frac{1}{\Omega} F\left(\mathbf{k}_{\mathbf{B}}+\mathbf{G}\right) e^{i\left(\mathbf{k}_{\mathbf{B}}+\mathbf{G}\right) \cdot \boldsymbol{\rho}},
$$

where the $\Omega=|\mathbf{a} \times \mathbf{b}|$ is the area of the unit cell of real $2 \mathrm{D}$ lattice, $\mathbf{G}$ are the displacement vectors of $2 \mathrm{D}$ reciprocallattice sites, and $F(\mathbf{k})$ is the Fourier transform of function $e^{i k_{\omega} r} / r$,

$$
\begin{gathered}
F(\mathbf{k})=\int d \boldsymbol{\rho} e^{-i \mathbf{k} \cdot \mathbf{r}} \frac{e^{i k_{\omega} r}}{r}=\frac{2 \pi i e^{i k_{z} z}}{k_{z}}, \\
k_{z}=\sqrt{k_{\omega}^{2}-k^{2}} .
\end{gathered}
$$

Here $\mathbf{r}=\boldsymbol{\rho}+z \hat{z}$ are $3 \mathrm{D}$ vectors, while $\boldsymbol{\rho}, \mathbf{R}, \mathbf{G}$, and $\mathbf{k}_{\mathbf{B}}$ are $2 \mathrm{D}$ vectors.

Putting Eqs. (A4) and (A3) into Eq. (A2), we have

$$
\begin{gathered}
S\left(k_{\omega}, \mathbf{k}_{\mathbf{B}}\right)=\lim _{z \rightarrow 0_{+}}\left(\frac{2 \pi i}{\Omega} \sum_{\mathbf{G}} \frac{e^{i k_{z}(\mathbf{G}) z}}{k_{z}(\mathbf{G})}-\frac{e^{i k_{\omega} z}}{z}\right), \\
k_{z}(\mathbf{G})=\sqrt{k_{\omega}^{2}-\left|\mathbf{k}_{\mathbf{B}}+\mathbf{G}\right|^{2}} .
\end{gathered}
$$

Given the case $\mathbf{k}_{\mathbf{B}}=0$, the real part of $S$ is found to have finite limit when $k_{\omega}$ and $z$ are tending to zero together. That limit can be defined as a constant,

$$
\begin{aligned}
D & =\operatorname{Re}\left[S\left(0_{+}, 0\right)\right]=\lim _{k_{\omega} \rightarrow 0_{+}} \operatorname{Re}\left(\sum_{\mathbf{R}} \frac{e^{i k_{\omega} R}}{R}\right) \\
& =\lim _{z \rightarrow 0_{+}}\left(\frac{2 \pi}{\Omega} \sum_{\mathbf{G}}^{\prime} \frac{e^{-z G}}{G}-\frac{1}{z}\right) .
\end{aligned}
$$

Adding to and subtracting from the right part of Eq. (A6) the value $D$ and taking Eq. (A8) into account, we have

$$
\begin{aligned}
S\left(k_{\omega}, \mathbf{k}_{\mathbf{B}}\right)= & D+\frac{2 \pi i}{\Omega} \frac{1}{\sqrt{k_{\omega}^{2}-k_{B}^{2}}}+\lim _{z \rightarrow 0_{+}}\left(\frac{1}{z}-\frac{e^{i k_{\omega} z}}{z}\right) \\
& +\frac{2 \pi}{\Omega} \lim _{z \rightarrow 0_{+}}\left[\sum_{\mathbf{G}}^{\prime} \frac{e^{-z \sqrt{\left|\mathbf{k}_{\mathbf{B}}+\mathbf{G}\right|^{2}-k_{\omega}^{2}}}}{\sqrt{\left|\mathbf{k}_{\mathbf{B}}+\mathbf{G}\right|^{2}-k_{\omega}^{2}}}-\frac{e^{-z G}}{G}\right] \\
= & D+\frac{2 \pi i}{\Omega} \frac{1}{\sqrt{k_{\omega}^{2}-k_{B}^{2}}}-i k_{\omega} \\
& +\frac{2 \pi}{\Omega} \sum_{\mathbf{G}}^{\prime}\left(\frac{1}{\sqrt{\left|\mathbf{k}_{\mathbf{B}}+\mathbf{G}\right|^{2}-k_{\omega}^{2}}}-\frac{1}{G}\right) .
\end{aligned}
$$

In Fig. 8, we compare the Simovski's series with a bruteforce real-space sum. While the original sum does not seem to converge, the Simovski's series quickly converges in a small circle with radius of a few hundred reciprocal-lattice constant. The convergence of the original sum becomes better in $k_{B}>\omega / c$ region than in $k_{B}<\omega / c$ because two neighboring dipoles are approximately antiphase when $k_{B}>\omega / c$ such that every two dipoles together form a quadruple of which the lattice sum has good convergence.

\section{APPENDIX B}

Let

$$
\begin{aligned}
\sum_{\mathbf{R} \neq \mathbf{0}} & \left(\frac{1}{R^{3}}-\frac{i k_{\omega}}{R^{2}}\right) e^{i\left(k_{\omega} R+\mathbf{k}_{\mathbf{B}} \cdot \mathbf{R}\right)} \\
= & \frac{1}{\Omega} \int_{R_{\min }}^{\infty}\left(\frac{1}{r^{2}}-\frac{i k_{\omega}}{r}\right) e^{i k_{\omega} r} d r \int_{0}^{2 \pi} e^{i k_{B} r \cos \theta} d \theta \\
= & \frac{2 \pi}{\Omega} \int_{R_{\min }}^{\infty}\left(\frac{1}{r^{2}}-\frac{i k_{\omega}}{r}\right) e^{i k_{\omega} r} J_{0}\left(k_{B} r\right) d r=\frac{2 \pi}{\Omega} I_{1}
\end{aligned}
$$

(here we also assume that the lattice sum is exactly equivalent to the integral from $R_{\min }$ to infinity, while $R_{\text {min }}$ is unknown initially), where

$$
\begin{aligned}
& I_{1}=\int_{R_{\min }}^{\infty}\left(\frac{1}{r^{2}}-\frac{i k_{\omega}}{r}\right) e^{i k_{\omega} r} J_{0}\left(k_{B} r\right) d r \\
&=-\int_{R_{\min }}^{\infty} J_{0}\left(k_{B} r\right) d \frac{e^{i k_{\omega} r}}{r} \\
&=\left.J_{0}\left(k_{B} r\right) \frac{e^{i k_{\omega} r}}{r}\right|_{\infty} ^{R_{\min }}+\int_{R_{\min }}^{\infty} \frac{e^{i k_{\omega} r}}{r} d J_{0}\left(k_{B} r\right) \\
&=\frac{J_{0}\left(k_{B} R_{\min }\right)}{R_{\min }} e^{i k_{\omega} R_{\min }}-k_{B}^{2} \int_{R_{\min }}^{\infty} \frac{J_{1}\left(k_{B} r\right)}{k_{B} r} e^{i k_{\omega} r} d r, \quad(\mathrm{~B} 2) \\
& \int_{R_{\min }}^{\infty} \frac{J_{1}\left(k_{B} r\right)}{k_{B} r} e^{i k_{\omega} r} d r=\frac{i}{k_{\omega}+\sqrt{k_{\omega}^{2}-k_{B}^{2}}}-\int_{0}^{R_{\min }} \frac{J_{1}\left(k_{B} r\right)}{k_{B} r} e^{i k_{\omega} r} d r,
\end{aligned}
$$

and

$$
\int_{0}^{R_{\min }} \frac{J_{1}\left(k_{B} r\right)}{k_{B} r} e^{i k_{\omega} r} d r \approx \frac{i}{2 k_{\omega}}\left(1-e^{i k_{\omega} R_{\min }}\right) .
$$

Finally we have

$$
\begin{aligned}
\therefore I_{1}= & \frac{J_{0}\left(k_{B} R_{\min }\right)}{R_{\min }} e^{i k_{\omega} R_{\min }} \\
& -k_{B}^{2}\left(\frac{i}{k_{\omega}+\sqrt{k_{\omega}^{2}-k_{B}^{2}}}-\int_{0}^{R_{\min }} \frac{J_{1}\left(k_{B} r\right)}{k_{B} r} e^{i k_{\omega} r} d r\right) \\
= & \frac{J_{0}\left(k_{B} R_{\min }\right)}{R_{\min }} e^{i k_{\omega} R_{\min }} \\
& -k_{B}^{2}\left[\frac{i}{k_{\omega}+\sqrt{k_{\omega}^{2}-k_{B}^{2}}}-\frac{i}{2 k_{\omega}}\left(1-e^{\left.i k_{\omega} R_{\min }\right)}\right] .\right.
\end{aligned}
$$

The parameter $R_{\min }$ can be determined in the electrostatic 

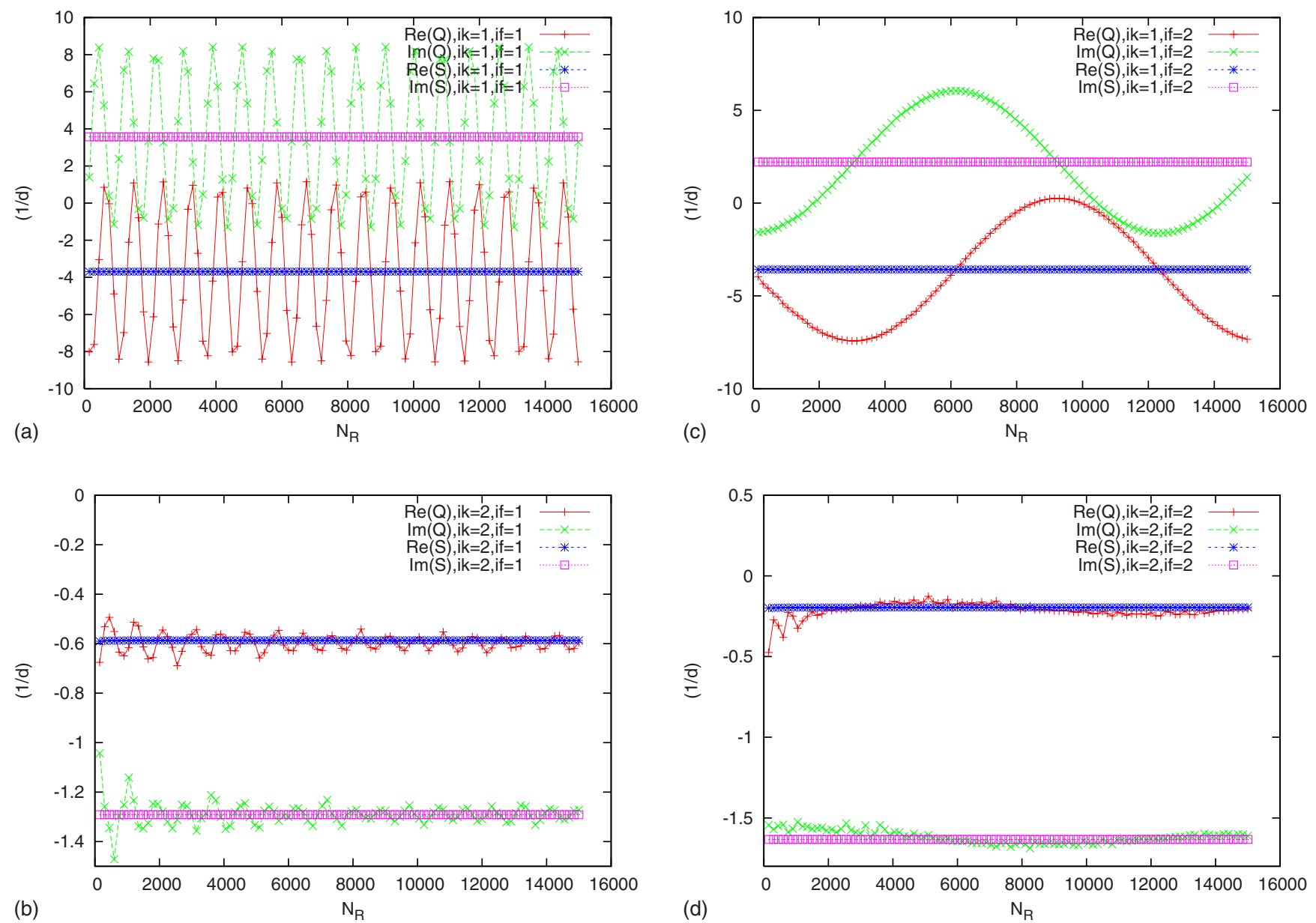

FIG. 8. (Color online) Examples of the convergence of the Simovski's series $(S)$ compared with the original real-lattice series $(Q)$. The circle within which the series is evaluated has a radius of $N_{R} d\left(2 \pi N_{R} / d\right)$ for original (Simovski) series. Lines with $+(\times)$ represent the real (imaginary) part of the original sum and lines with stars (open squares) represent the real (imaginary) part of the Simovski's series. The denotation "if" $=1,2$ are for $\omega<\omega_{0}, \omega>\omega_{0}$ and "ik" $=1,2$ are for $k_{B}<\omega / c, k_{B}>\omega / c$, respectively. (a) $\omega<\omega_{0}$ and $k_{B}<\omega / c$; (b) $\omega<\omega_{0}$ and $k_{B}>\omega / c$; (c) $\omega>\omega_{0}$ and $k_{B}<\omega / c$; and (d) $\omega>\omega_{0}$ and $k_{B}>\omega / c$.

limit by making $k_{B}=0$ and $k_{\omega} \rightarrow 0$. Then we have

$$
\sum_{\mathbf{R} \neq \mathbf{0}} \frac{1}{R^{3}}=\frac{2 \pi}{\Omega} \lim _{k_{\omega} \rightarrow 0} I_{1}\left(k_{\omega}, 0\right)=\frac{2 \pi}{\Omega} \frac{1}{R_{\min }} .
$$

For square lattice, the numerical result is $R_{\min }=d / 1.438$.

Similarly, let

$$
\begin{aligned}
\sum_{\mathbf{R} \neq \mathbf{0}}\left(\frac{k_{\omega}^{2}}{R}\right) e^{i\left(k_{\omega} R+\mathbf{k}_{\mathbf{B}} \cdot \mathbf{R}\right)} & \\
= & \frac{1}{\Omega} \int_{R_{\min }}^{\infty} k_{\omega}^{2} e^{i k_{\omega} r} d r \int_{0}^{2 \pi} e^{i k_{B} r \cos \theta} d \theta \\
= & \frac{2 \pi}{\Omega} \int_{R_{\min }}^{\infty} k_{\omega}^{2} e^{i k_{\omega^{r}} J_{0}}\left(k_{B} r\right) d r=\frac{2 \pi}{\Omega} I_{2},
\end{aligned}
$$

$$
\begin{aligned}
I_{2} & =\int_{R_{\min }}^{\infty} k_{\omega}^{2} e^{i k_{\omega} r} J_{0}\left(k_{B} r\right) d r=-i k_{\omega} \int_{R_{\min }}^{\infty} J_{0}\left(k_{B} r\right) d\left(e^{i k_{\omega} r}\right) \\
& =-\left.i k_{\omega} J_{0}\left(k_{B} r\right) e^{i k_{\omega} r}\right|_{R_{\min }} ^{\infty}+i k_{\omega} \int_{R_{\min }}^{\infty} e^{i k_{\omega} r} d\left[J_{0}\left(k_{B} r\right)\right] \\
& =i k_{\omega}\left[J_{0}\left(k_{B} R_{\min }\right) e^{i k_{\omega} R_{\min }}-k_{B} \int_{R_{\min }}^{\infty} J_{1}\left(k_{B} r\right) e^{i k_{\omega} r} d r\right] \\
& \int_{R_{\min }}^{\infty} J_{1}\left(k_{B} r\right) e^{i k_{\omega} r} d r=\frac{-k_{B}}{\sqrt{k_{\omega}^{2}-k_{B}^{2}}\left(k_{\omega}+\sqrt{k_{\omega}^{2}-k_{B}^{2}}\right)} \\
& -\int_{0}^{R_{\min }} J_{1}\left(k_{B} r\right) e^{i k_{\omega} r} d r
\end{aligned}
$$

where 


$$
\int_{0}^{R_{\min }} J_{1}\left(k_{B} r\right) e^{i k_{\omega} r} d r \approx \frac{k_{B}}{2 k_{\omega}^{2}}\left[\left(1-i k_{\omega} R_{\min }\right) e^{i k_{\omega} R_{\min }}-1\right] .
$$

In Eqs. (B4) and (B10) the integrands are approximated as $J_{1}\left(k_{B} r\right) / k_{B} r \approx 1 / 2$ and $J_{1}\left(k_{B} r\right) \approx k_{B} r / 2$, the same as those in Ref. 20. Therefore

$$
\beta_{\mathrm{T}}=\sum_{\mathbf{R} \neq \mathbf{0}}\left(-\frac{1}{R^{3}}+\frac{i k_{\omega}}{R^{2}}+\frac{k_{\omega}^{2}}{R}\right) e^{i\left(k_{\omega} R+\mathbf{k}_{\mathrm{B}} \cdot \mathbf{R}\right)}=\frac{2 \pi}{\Omega}\left(I_{2}-I_{1}\right) .
$$

By approximating the lattice sum for dense dipole array as integrals, we have

$$
\begin{aligned}
\beta_{\mathrm{LI}}= & \frac{1}{\Omega} \int_{R_{\min }}^{\infty} \int_{0}^{2 \pi} e^{i k_{B} r \cos \theta} e^{i k_{\omega}{ }^{r}}\left[\left(3 \cos ^{2} \theta-1\right)\left(\frac{1}{r^{2}}-\frac{i k_{\omega}}{r}\right)\right. \\
& \left.+k_{\omega}^{2} \sin ^{2} \theta\right] d r d \theta,
\end{aligned}
$$

$$
\begin{aligned}
\beta_{\mathrm{TI}}= & \frac{1}{\Omega} \int_{R_{\min }}^{\infty} \int_{0}^{2 \pi} e^{i k_{B} r \cos \theta} e^{i k_{\omega} r}\left[\left(3 \sin ^{2} \theta-1\right)\left(\frac{1}{r^{2}}-\frac{i k_{\omega}}{r}\right)\right. \\
& \left.+k_{\omega}^{2} \cos ^{2} \theta\right] d r d \theta .
\end{aligned}
$$

From Ref. 20, we know that $\beta_{\mathrm{LI}}=2 \pi\left(I_{1}-I_{3}\right) / \Omega$ and $\beta_{\mathrm{TI}}$ $=2 \pi\left(I_{2}+I_{3}\right) / \Omega$, where

$$
I_{3}=\left(\frac{1}{R_{\min }}-i k_{\omega}\right) \frac{J_{1}\left(k_{B} R_{\min }\right)}{k_{B} R_{\min }} e^{i k_{\omega} R_{\min }}
$$

Then for $k_{B}<k_{\omega}$ cases, we have

$$
\begin{aligned}
\operatorname{Re}\left[I_{1}\right] & =\frac{J_{0}\left(k_{B} R_{\min }\right)}{R_{\min }} \cos \left(k_{\omega} R_{\min }\right)+\frac{k_{B}^{2}}{2 k_{\omega}} \operatorname{Re}\left[i\left(1-e^{\left.i k_{\omega} R_{\min }\right)}\right]\right. \\
& =\frac{J_{0}\left(k_{B} R_{\min }\right)}{R_{\min }} \cos \left(k_{\omega} R_{\min }\right)+\frac{k_{B}^{2}}{2 k_{\omega}} \sin \left(k_{\omega} R_{\min }\right), \quad(\mathrm{B} 15)
\end{aligned}
$$

$$
\begin{aligned}
\operatorname{Re}\left[I_{2}\right] & =-k_{\omega} \operatorname{Im}\left[J_{0}\left(k_{B} R_{\min }\right) e^{i k_{\omega} R_{\min }}-k_{B} \int_{R_{\min }}^{\infty} J_{1}\left(k_{B} r\right) e^{i k_{\omega} r} d r\right] \\
& =-k_{\omega}\left\{J_{0}\left(k_{B} R_{\min }\right) \sin \left(k_{\omega} R_{\min }\right)-k_{B} \operatorname{Im}\left[\frac{-k_{B}}{\sqrt{k_{\omega}^{2}-k_{B}^{2}}\left(k_{\omega}+\sqrt{k_{\omega}^{2}-k_{B}^{2}}\right)}-\int_{0}^{R_{\min }} J_{1}\left(k_{B} r\right) e^{i k_{\omega} r} d r\right]\right\} \\
& =-k_{\omega}\left\{J_{0}\left(k_{B} R_{\min }\right) \sin \left(k_{\omega} R_{\min }\right)+\frac{k_{B}^{2}}{2 k_{\omega}^{2}} \operatorname{Im}\left[\left(1-i k_{\omega} R_{\min }\right) e^{\left.i k_{\omega} R_{\min }-1\right]}\right\}\right. \\
& =-k_{\omega}\left\{J_{0}\left(k_{B} R_{\min }\right) \sin \left(k_{\omega} R_{\min }\right)+\frac{k_{B}^{2}}{\left.2 k_{\omega}^{2}\left[\sin \left(k_{\omega} R_{\min }\right)-k_{\omega} R_{\min } \cos \left(k_{\omega} R_{\min }\right)\right]\right\},}\right. \\
\operatorname{Re}\left[I_{3}\right] & =\frac{J_{1}\left(k_{B} R_{\min }\right)}{k_{B} R_{\min }} \operatorname{Re}\left[\left(\frac{1}{R_{\min }}-i k_{\omega}\right) e^{i k_{\omega} R_{\min }}\right]=\frac{J_{1}\left(k_{B} R_{\min }\right)}{k_{B} R_{\min }}\left[\frac{\cos \left(k_{\omega} R_{\min }\right)+k_{\omega} R_{\min } \sin \left(k_{\omega} R_{\min }\right)}{R_{\min }}\right] .
\end{aligned}
$$

Noting that the Bessel functions $J_{0}$ and $J_{1}$ in the integrals can be expanded in polynomials for small arguments as $J_{0}(x) \sim 1$ $-x^{2} / 4+O\left(x^{4}\right)$ and $J_{1}(x) / x \sim 1 / 2-x^{2} / 16+O\left(x^{4}\right)$ and we have $k_{B} R_{\min } \ll 1$ inside the light cone, the form of $\operatorname{Re}\left[\beta_{\sigma}\right]$ near resonant frequencies $\omega_{\sigma}$ can be approximated in

$$
\begin{aligned}
\operatorname{Re}\left[\beta_{\mathrm{T}}\right] & =\frac{2 \pi}{\Omega}\left\{-\frac{k_{B}^{2}}{2 k_{\omega}}\left[2 \sin \left(k_{\omega} R_{\min }\right)-k_{\omega} R_{\min } \cos \left(k_{\omega} R_{\min }\right)\right]-\frac{J_{0}\left(k_{B} R_{\min }\right)}{R_{\min }}\left[\cos \left(k_{\omega} R_{\min }\right)+k_{\omega} R_{\min } \sin \left(k_{\omega} R_{\min }\right)\right]\right\} \\
& \approx \frac{2 \pi}{\Omega}\left\{-\frac{k_{B}^{2}}{2 k_{\omega}}\left[2 \sin \left(k_{\omega} R_{\min }\right)-k_{\omega} R_{\min } \cos \left(k_{\omega} R_{\min }\right)\right]-\frac{4-\left(k_{B} R_{\min }\right)^{2}}{4 R_{\min }}\left[\cos \left(k_{\omega} R_{\min }\right)+k_{\omega} R_{\min } \sin \left(k_{\omega} R_{\min }\right)\right]\right\} \\
& \approx \frac{2 \pi}{\Omega}\left[-\frac{\cos \left(k_{\omega} R_{\min }\right)+k_{\omega} R_{\min } \sin \left(k_{\omega} R_{\min }\right)}{R_{\min }}+k_{B}^{2} R_{\min }^{2} \frac{3 \cos \left(k_{\omega} R_{\min }\right)-3 \sin \left(k_{\omega} R_{\min }\right)}{4 R_{\min }}\right],
\end{aligned}
$$




$$
\begin{aligned}
& \operatorname{Re}\left[\beta_{\mathrm{TI}}\right]= \frac{2 \pi}{\Omega}\left\{\frac{J_{1}\left(k_{B} R_{\min }\right)}{k_{B} R_{\min }}\left[\frac{\cos \left(k_{\omega} R_{\min }\right)+k_{\omega} R_{\min } \sin \left(k_{\omega} R_{\min }\right)}{R_{\min }}\right]-J_{0}\left(k_{B} R_{\min }\right) k_{\omega} \sin \left(k_{\omega} R_{\min }\right)-\frac{k_{B}^{2}}{2 k_{\omega}}\left[\sin \left(k_{\omega} R_{\min }\right)\right.\right. \\
&\left.\left.-k_{\omega} R_{\min } \cos \left(k_{\omega} R_{\min }\right)\right]\right\} \approx \frac{2 \pi}{\Omega}\left\{\left(\frac{1}{2}-\frac{k_{B}^{2} R_{\min }^{2}}{16}\right)\left[\frac{\cos \left(k_{\omega} R_{\min }\right)+k_{\omega} R_{\min } \sin \left(k_{\omega} R_{\min }\right)}{R_{\min }}\right]\right. \\
&\left.-\left(1-\frac{k_{B}^{2} R_{\min }^{2}}{4}\right) \frac{k_{\omega} R_{\min } \sin \left(k_{\omega} R_{\min }\right)}{R_{\min }}-\frac{k_{B}^{2} R_{\min }^{2}}{2 k_{\omega} R_{\min }}\left[\frac{\sin \left(k_{\omega} R_{\min }\right)-k_{\omega} R_{\min } \cos \left(k_{\omega} R_{\min }\right)}{R_{\min }}\right]\right\} \\
& \approx \frac{2 \pi}{\Omega}\left[\frac{\cos \left(k_{\omega} R_{\min }\right)-k_{\omega} R_{\min } \sin \left(k_{\omega} R_{\min }\right)}{2 R_{\min }}+k_{B}^{2} R_{\min }^{2} \frac{7 \cos \left(k_{\omega} R_{\min }\right)-5 \sin \left(k_{\omega} R_{\min }\right)}{16 R_{\min }}\right], \\
&\left.\operatorname{Re} \beta_{\mathrm{LI}}\right]=\frac{2 \pi}{\Omega}\left\{\frac{J_{0}\left(k_{B} R_{\min }\right)}{\left.R_{\min } \cos \left(k_{\omega} R_{\min }\right)+\frac{k_{B}^{2}}{2 k_{\omega}} \sin \left(k_{\omega} R_{\min }\right)-\frac{J_{1}\left(k_{B} R_{\min }\right)}{k_{B} R_{\min }}\left[\frac{\cos \left(k_{\omega} R_{\min }\right)+k_{\omega} R_{\min } \sin \left(k_{\omega} R_{\min }\right)}{R_{\min }}\right]\right\}}\right. \\
& \approx \frac{2 \pi}{\Omega}\left\{\frac{4-\left(k_{B} R_{\min }\right)^{2}}{4 R_{\min }} \cos \left(k_{\omega} R_{\min }\right)+\frac{k_{B}^{2} R_{\min }^{2}}{2 k_{\omega} R_{\min }} \frac{\sin \left(k_{\omega} R_{\min }\right)}{R_{\min }}-\left(\frac{1}{2}-\frac{k_{B}^{2} R_{\min }^{2}}{16}\right)\left[\frac{\cos \left(k_{\omega} R_{\min }\right)+k_{\omega} R_{\min } \sin \left(k_{\omega} R_{\min }\right)}{R_{\min }}\right]\right\} \\
& \approx \frac{2 \pi}{\Omega}\left[\frac{\cos \left(k_{\omega} R_{\min }\right)-k_{\omega} R_{\min } \sin \left(k_{\omega} R_{\min }\right)}{2 R_{\min }}+k_{B}^{2} R_{\min }^{2} \frac{-3 \cos \left(k_{\omega} R_{\min }\right)+9 \sin \left(k_{\omega} R_{\min }\right)}{16 R_{\min }}\right],
\end{aligned}
$$

where we have taken the approximation $k_{\omega} R_{\min }=\omega R_{\min } / c \approx \omega_{0} R_{\min } / c \approx 1$ near resonance, in order to simplify the coefficient associated with the quadratic term $k_{B}^{2} R_{\min }^{2}$, and such simplified coefficient can make it easier to compare between different modes.

${ }^{1}$ M. Quinten, A. Leitner, J. R. Krenn, and F. R. Aussenegg, Opt. Lett. 23, 1331 (1998).

${ }^{2}$ M. L. Brongersma, J. W. Hartman, and H. A. Atwater, Phys. Rev. B 62, R16356 (2000).

${ }^{3}$ S. A. Maier, M. L. Brongersma, P. G. Kik, S. Meltzer, A. A. G. Requicha, and H. A. Atwater, Adv. Mater. (Weinheim, Ger.) 13, 1501 (2001).

${ }^{4}$ S. A. Maier, P. G. Kik, H. A. Atwater, S. Meltzer, E. Harel, B. E. Koel, and A. A. G. Requicha, Nat. Mater. 2, 229 (2003).

${ }^{5}$ S. A. Maier and H. A. Atwater, J. Appl. Phys. 98, 011101 (2005).

${ }^{6}$ R. Elghanian, J. J. Storhoff, R. C. Mucic, R. L. Letsinger, and C. A. Mirkin, Science 277, 1078 (1997).

${ }^{7}$ A. J. Haes, S. Zou, G. C. Schatz, and R. P. Van Duyne, J. Phys. Chem. B 108, 6961 (2004); A. J. Haes and R. P. Van Duyne, J. Am. Chem. Soc. 124, 10596 (2002).

${ }^{8}$ C. R. Simovski, A. J. Viitanen, and S. A. Tretyakov, Phys. Rev. E 72, 066606 (2005).

${ }^{9}$ P. Alitalo, C. R. Simovski, A. Viitanen, and S. Tretyakov, Phys. Rev. B 74, 235425 (2006).

${ }^{10}$ S. Y. Park and D. Stroud, Phys. Rev. B 69, 125418 (2004).

${ }^{11}$ W. H. Weber and G. W. Ford, Phys. Rev. B 70, 125429 (2004).

${ }^{12}$ D. S. Citrin, Nano Lett. 4, 1561 (2004).

${ }^{13}$ R. A. Shore and A. D. Yaghjian, Electron. Lett. 41, 578 (2005).

${ }^{14}$ R. A. Shore and A. D. Yaghjian, Radio Sci. 42, RS6S21 (2007).

${ }^{15}$ A. F. Koenderink and A. Polman, Phys. Rev. B 74, 033402 (2006).

${ }^{16}$ K. H. Fung and C. T. Chan, Opt. Lett. 32, 973 (2007).

${ }^{17}$ C. L. Haynes, A. D. McFarland, L. Zhao, R. P. Van Duyne, G. C. Schatz, L. Gunnarsson, J. Prikulis, B. Kasemo, and M. Kall, J. Phys. Chem. B 107, 7337 (2003).
${ }^{18}$ J. Cesario, R. Quidant, G. Badenes, and S. Enoch, Opt. Lett. 30, 3404 (2005).

${ }^{19}$ C. R. Simovski, P. A. Belov, and M. S. Kondratjev, J. Electromagn. Waves Appl. 13, 189 (1999).

${ }^{20}$ A. J. Viitanen, I. Hänninen, and S. A. Tretyakov, PIER 38, 97 (2002).

${ }^{21}$ L. Zhao, K. L. Kelly, and G. C. Schatz, J. Phys. Chem. B 107, 7343 (2003).

${ }^{22}$ S. Zou and G. C. Schatz, J. Chem. Phys. 121, 12606 (2004).

${ }^{23}$ D. S. Citrin, Opt. Lett. 31, 98 (2006).

${ }^{24}$ V. A. Markel and V. M. Shalaev, in Computational Studies of New Materials, edited by D. A. Jelski and T. F. George (World Scientific, Singapore, 1999).

${ }^{25}$ L. Zhou and S. T. Chui, Phys. Rev. B 74, 035419 (2006).

${ }^{26}$ S. A. Maier, P. G. Kik, and H. A. Atwater, Phys. Rev. B 67, 205402 (2003).

${ }^{27}$ J. D. Jackson, Classical Electrodynamics, 3rd ed. (Wiley, New York, 1998).

${ }^{28}$ See, e.g., A. Wokaun, J. P. Gordon, and P. F. Liao, Phys. Rev. Lett. 48, 957 (1982).

${ }^{29} \mathrm{Jack} \mathrm{Ng}$ (private communication).

${ }^{30}$ B. T. Draine, Astron. J. 333, 848 (1988).

${ }^{31}$ P. A. Belov, C. R. Simovski, and M. S. Kondratjev, Proc. SPIE 3039, 680 (1997).

${ }^{32}$ L. Tsang, J. A. Kong, K. H. Ding, and C. O. Ao, Scattering of Electromagnetic Waves: Numerical Simulations (Wiley, New York, 2001).

${ }^{33}$ C. R. Simovski (private communication).

${ }^{34}$ P. B. Johnson and R. W. Christy, Phys. Rev. B 6, 4370 (1972).

${ }^{35}$ J. J. Xiao, J. P. Huang, and K. W. Yu, Phys. Rev. B 71, 045404 (2005). 